\title{
Eclectic flavor groups
}

\author{
Hans Peter Nilles, ${ }^{a}$ Saúl Ramos-Sánchez ${ }^{b, c}$ and Patrick K.S. Vaudrevange ${ }^{c}$ \\ ${ }^{a}$ Bethe Center for Theoretical Physics and Physikalisches Institut der Universität Bonn, \\ Nussallee 12, 53115 Bonn, Germany \\ ${ }^{b}$ Instituto de Física, Universidad Nacional Autónoma de México, \\ POB 20-364, Cd.Mx. 01000, México \\ ${ }^{c}$ Physik Department T75, Technische Universität München, \\ James-Franck-Straße 1, 85748 Garching, Germany \\ E-mail: nilles@th.physik.uni-bonn.de, ramos@fisica.unam.mx, \\ patrick. vaudrevange@tum.de
}

ABSTRACT: The simultaneous study of top-down and bottom-up approaches to modular flavor symmetry leads necessarily to the concept of eclectic flavor groups. These are nontrivial products of modular and traditional flavor symmetries that exhibit the phenomenon of local flavor enhancement in moduli space. We develop methods to determine the eclectic flavor groups that can be consistently associated with a given traditional flavor symmetry. Applying these methods to a large family of prominent traditional flavor symmetries, we try to identify potential candidates for realistic eclectic flavor groups and show that they are relatively rare. Model building with finite modular flavor symmetries thus appears to be much more restrictive than previously thought.

Keywords: Beyond Standard Model, Discrete Symmetries, Global Symmetries, Quark Masses and SM Parameters

ArXiv ePrint: 2001.01736 


\section{Contents}

1 Introduction 1

2 Extending flavor symmetries by modular symmetries $\quad 3$

$\begin{array}{lll}2.1 & \text { The eclectic extension } & 6\end{array}$

$\begin{array}{ll}2.2 \text { Combining with } \mathcal{C P} & 7\end{array}$

$\begin{array}{llr}3 & \text { Local flavor unification } & 9\end{array}$

4 Example: $\Delta(54)$ and modular symmetries 10

$\begin{array}{llr}5 & \text { Results } & 12\end{array}$

6 Conclusions 13

$\begin{array}{ll}\text { A Remarks } & 15\end{array}$

B Explicit examples $\quad \mathbf{1 5}$

$\begin{array}{lll}\text { B.1 Traditional flavor symmetry } Q_{8} & 15\end{array}$

B.2 Traditional flavor symmetry $\mathbb{Z}_{3} \times \mathbb{Z}_{3} \quad 16$

$\begin{array}{lll}\text { B.3 Traditional flavor symmetry } A_{4} & 17\end{array}$

$\begin{array}{lll}\text { B.4 Traditional flavor symmetry } T^{\prime} & 18\end{array}$

$\begin{array}{ll}\text { B.5 Traditional flavor symmetry } \Delta(27) & 18\end{array}$

B.6 Trivial extension of the traditional flavor symmetry $\Delta(54) \quad 20$

\section{Introduction}

While the parameters of the flavor sector of the Standard Model of particle physics have essentially all been determined experimentally, the origin of "flavor" remains a mystery. Models with traditional (discrete) flavor symmetries have provided various fits for masses and mixing angles of quarks and leptons [1]. More recently, it was suggested that (discrete) modular symmetries might describe the flavor structure of the lepton sector [2]. This bottom-up (BU) description is based on the consideration of finite modular groups $\Gamma_{N}$ with $N=2,3,4,5$. Typically, in these models, some of the lepton multiplets are described by nontrivial singlets or irreducible triplets of $\Gamma_{N}$. This suggestion has an important impact on the field of lepton flavor physics [3-41].

To understand the origin of flavor and modular symmetries we need to consider additionally a top-down (TD) approach, based on ultraviolet complete theories. Recently, such attempts have studied modular symmetries in string derived standard-like models based on 
heterotic orbifolds [42, 43] and magnetized D-branes [44-47] in connection with the standard discrete flavor symmetries within this framework [48]. This leads to a hybrid picture where the traditional flavor group and the finite modular group combine (as a nontrivial product $^{1}$ ) to a generalized flavor group which we call "Eclectic Flavor Group". It contains the traditional flavor group (which acts universally in moduli space) as well as the corresponding modular flavor structure $\Gamma_{N}$. This picture includes the mechanism of "Local Flavor Unification" of flavor, $\mathcal{C P}$ and modular symmetries with enhanced symmetries at certain locations in moduli space [42, 43], see also ref. [34]. Furthermore, it potentially incorporates a different flavor structure for the quark- and lepton-sector of the Standard Model.

In the present paper we want to analyze possible relations between the bottom-up (BU) and the top-down (TD) approaches. At first sight, we are confronted with some potential obstructions. First of all, it seems to be difficult to find nontrivial singlets and irreducible triplet representations of finite modular groups within the TD approach. Such triplets, as used in the BU-case, can be identified more easily for traditional flavor groups, as e.g. in $\Delta(54)$ of ref. [48]. In addition, the TD picture does not always lead to $\Gamma_{N}$ itself (as the finite modular group), but to its double cover ( $T^{\prime}$ in case of $\Gamma_{3}$, see refs. [42, 43]). Although we have studied up to now only a limited number of TD-models, we can emphasize the following key observation:

The full eclectic flavor group is a nontrivial product of the traditional flavor group, a corresponding finite modular group and a $\mathcal{C} \mathcal{P}$-like transformation. We cannot treat these symmetries separately (as mostly done in the BU-approach) and have to be aware of restrictions (for superpotential and Kähler potential [33]) from all of these components.

As a step in our search for a connection between BU- and TD-approaches, we shall develop a classification method to obtain all allowed eclectic flavor groups. This is the main goal of the present paper. We shall show that this combination of traditional flavor group and finite modular group cannot be arbitrary but has to satisfy severe consistency conditions. This can be seen already from the results of previous work [42, 43], where it was observed that candidate eclectic flavor groups derive from the traditional flavor group and its outer automorphisms. ${ }^{2}$ Based on this observation, we shall classify the possible eclectic flavor groups in a bottom-up way (for a class of prominent traditional flavor groups) and show that there is only a limited number of possibilities.

The paper is structured as follows. In section 2 we shall discuss the interplay of traditional flavor and modular symmetries and derive relevant consistency conditions. Section 3 discusses the question of "Local Flavor Unification" from the point of view of allowed modular symmetries. In section 4 we give a specific example (closely related to the $\mathbb{T}^{2} / \mathbb{Z}_{3}$ orbifold) with traditional flavor group $\Delta(54)$ and its eclectic extension by $T^{\prime}$ (being the double cover of $\Gamma_{3}$ ). Other explicit examples are relegated to the appendices, where we also show that there is no eclectic extension of $\Delta(54)$ with $\Gamma_{2}$ (as a result of the consistency conditions derived in section 2). Our results are displayed in table 2, where one can read off

\footnotetext{
${ }^{1}$ In the TD approach, the traditional flavor group does not commute with the finite modular group.

${ }^{2}$ This is connected to the outer automorphisms of the Narain space group [49] as explained in refs. [42, 43].
} 


\begin{tabular}{|c|cl|cl|cl|cl|}
\hline level & \multicolumn{4}{|c|}{ without $\mathcal{C P}$} & \multicolumn{4}{c|}{ with $\mathcal{C P}$} \\
$N$ & $\Gamma_{N}$ & $\mathrm{GAP}$ & $\Gamma_{N}^{\prime}$ & $\mathrm{GAP}$ & $\Gamma_{N}^{*}$ & $\mathrm{GAP}$ & $\Gamma_{N}^{\prime *}$ & $\mathrm{GAP}$ \\
\hline 2 & $S_{3}$ & {$[6,1]$} & $S_{3}$ & {$[6,1]$} & $S_{3} \times \mathbb{Z}_{2}$ & {$[12,4]$} & $S_{3} \times \mathbb{Z}_{2}$ & {$[12,4]$} \\
3 & $A_{4}$ & {$[12,3]$} & $T^{\prime}$ & {$[24,3]$} & $S_{4}$ & {$[24,12]$} & $\mathrm{GL}(2,3)$ & {$[48,29]$} \\
4 & $S_{4}$ & {$[24,12]$} & $\mathrm{SL}(2,4)$ & {$[48,30]$} & $S_{4} \times \mathbb{Z}_{2}$ & {$[48,48]$} & $\mathrm{GL}\left(2, \mathbb{Z}_{4}\right)$ & {$[96,195]$} \\
5 & $A_{5}$ & {$[60,5]$} & $\mathrm{SL}(2,5)$ & {$[120,5]$} & $A_{5} \times \mathbb{Z}_{2}$ & {$[120,35]$} & $\mathrm{SL}(2,5) \rtimes \mathbb{Z}_{2}$ & {$[240,93]$} \\
\hline
\end{tabular}

Table 1. Overview of finite modular groups without and with a $\mathcal{C P}$-like transformation (see section 2.2 for the details on $\mathcal{C P}$ ). The column GAP labels the groups according to ref. [50], where the first number gives the order of the group. We remark several group isomorphisms: $\Gamma_{2} \cong \Gamma_{2}^{\prime} \cong S_{3}$, $\Gamma_{2}^{*} \cong \Gamma_{2}^{* *} \cong S_{3} \times \mathbb{Z}_{2} \cong D_{12}, \mathrm{SL}(2,2) \cong S_{3}$ and $\mathrm{SL}(2,3) \cong T^{\prime}$

the allowed eclectic flavor groups for representative examples of several traditional flavor groups. Section 6 gives conclusions and outlook.

\section{Extending flavor symmetries by modular symmetries}

The modular group $\operatorname{SL}(2, \mathbb{Z})$ can be defined by the presentation $[51]^{3}$

$$
\mathrm{SL}(2, \mathbb{Z})=\left\langle\mathrm{S}, \mathrm{T} \mid \mathrm{S}^{4}=\mathbb{1},(\mathrm{ST})^{3}=\mathbb{1}, \mathrm{S}^{2} \mathrm{~T}=\mathrm{TS}^{2}\right\rangle,
$$

and a choice of $\operatorname{SL}(2, \mathbb{Z})$ generators $\mathrm{S}$ and $\mathrm{T}$ is given by

$$
\mathrm{S}=\left(\begin{array}{cc}
0 & 1 \\
-1 & 0
\end{array}\right) \quad \text { and } \quad \mathrm{T}=\left(\begin{array}{ll}
1 & 1 \\
0 & 1
\end{array}\right)
$$

Under a modular transformation $\gamma \in \mathrm{SL}(2, \mathbb{Z})$ both, the modulus $\tau$ and matter fields $\psi$, transform in general nontrivially according to

$$
\tau \stackrel{\gamma}{\longmapsto} \frac{a \tau+b}{c \tau+d}, \quad \psi \stackrel{\gamma}{\longmapsto}(c \tau+d)^{-k} \rho(\gamma) \psi \quad \text { for } \quad \gamma=\left(\begin{array}{ll}
a & b \\
c & d
\end{array}\right) \in \operatorname{SL}(2, \mathbb{Z}) .
$$

Here, $k \in \mathbb{Q}$ is the so-called modular weight ${ }^{4}$ of $\psi$ and $\rho\left(\gamma_{1} \gamma_{2}\right)=\rho\left(\gamma_{1}\right) \rho\left(\gamma_{2}\right)$ is a representation of the finite modular group $\Gamma_{N}$ or of its double cover $\Gamma_{N}^{\prime}[22]$ for $N \in\{2,3,4,5\}$. These finite groups are defined by the presentations

$$
\begin{aligned}
& \Gamma_{N}=\left\langle\mathrm{S}, \mathrm{T} \mid \mathrm{S}^{2}=\mathbb{1},(\mathrm{ST})^{3}=\mathbb{1}, \mathrm{T}^{N}=\mathbb{1}\right\rangle \\
& \Gamma_{N}^{\prime}=\left\langle\mathrm{S}, \mathrm{T} \mid \mathrm{S}^{4}=\mathbb{1},(\mathrm{ST})^{3}=\mathbb{1}, \mathrm{T}^{N}=\mathbb{1}, \mathrm{S}^{2} \mathrm{~T}=\mathrm{TS}^{2}\right\rangle
\end{aligned}
$$

All finite modular groups $\Gamma_{N}$ and $\Gamma_{N}^{\prime}$ with $N=2,3,4,5$ are listed in table 1.

\footnotetext{
${ }^{3}$ See page 81 using $S=S_{\text {Serre }}$ and $T=S_{\text {Serre }} T_{\text {Serre }}^{-1} S_{\text {Serre }}^{3}$

${ }^{4}$ In contrast to modular forms $Y(\tau)$, fields can have fractional weights as realized in string theory [52-54].
} 
The symmetry group of a modular invariant theory is $\mathrm{SL}(2, \mathbb{Z})$. This symmetry has different realizations for the various fields of the theory: the $\tau$ modulus feels only $\operatorname{PSL}(2, \mathbb{Z})$ since $\pm \gamma \in \mathrm{SL}(2, \mathbb{Z})$ yield the same transformation (2.3) for $\tau$. In contrast, a matter field $\psi$ transforms twofold:

i) by the automorphy factor $(c \tau+d)^{-k}$ that distinguishes between $\gamma$ and $-\gamma$ for general modular weight $k$, and

ii) by a linear transformation $\rho(\gamma)$ that can distinguish between $\gamma$ and $-\gamma$ only in the case $\Gamma_{N}^{\prime}$ with $N>2$, where $\rho(\gamma) \neq \rho(-\gamma)$ if $\rho(-\mathbb{1})=\rho\left(\mathrm{S}^{2}\right) \neq \mathbb{1}$ is nontrivial.

Finally, due to the transformation of the $\tau$ modulus, Yukawa couplings $Y(\tau)$ are in general modular forms and transform similar to eq. (2.3) as

$$
Y(\tau) \stackrel{\gamma}{\longmapsto} Y\left(\frac{a \tau+b}{c \tau+d}\right)=(c \tau+d)^{k_{Y}} \rho_{Y}(\gamma) Y(\tau) \quad \text { for } \quad \gamma=\left(\begin{array}{ll}
a & b \\
c & d
\end{array}\right) \in \operatorname{SL}(2, \mathbb{Z}),
$$

where $\rho_{Y}(\gamma)$ is also a representation of the finite modular group. For a given modular weight $k_{Y}$ (with $k_{Y} \in 2 \mathbb{N}$ for $\Gamma_{N}$ or $k_{Y} \in \mathbb{N}$ for $\Gamma_{N}^{\prime}$ ), the number of independent Yukawa couplings $Y(\tau)$ is finite and their $\tau$-dependence and transformations $\rho_{Y}(\gamma)$ are explicitly known, see e.g. $[2,5,6,10,15,22,40]$. Hence, in order to fully specify a modular invariant theory one has to choose the finite modular group $\Gamma_{N}$ or $\Gamma_{N}^{\prime}$ that shall host the representation matrices $\rho(\gamma)$ and $\rho_{Y}(\gamma)$.

Now, since the matrices $\rho(\gamma)$ in eq. (2.3) and $\rho_{Y}(\gamma)$ in eq. (2.5) must build a (reducible or irreducible) representation of $\Gamma_{N}$ or $\Gamma_{N}^{\prime}$, they have to satisfy the respective presentation $(2.4 \mathrm{a})$ or $(2.4 \mathrm{~b})$, i.e.

$$
(\rho(\mathrm{S}))^{N_{\mathrm{S}}}=\mathbb{1}, \quad(\rho(\mathrm{T}))^{N}=\mathbb{1}, \quad(\rho(\mathrm{S} \mathrm{T}))^{3}=\mathbb{1}, \quad \text { and } \quad \rho\left(\mathrm{S}^{2} \mathrm{~T}\right)=\rho\left(\mathrm{T} \mathrm{S}^{2}\right),
$$

with $N \in\{2,3,4,5\}$ and $N_{\mathrm{S}}=2$ for $\Gamma_{N}$ or $N_{\mathrm{S}}=4$ for $\Gamma_{N}^{\prime}$.

Let us stress that the $\tau$ modulus transforms nontrivially under modular transformations eq. (2.3). In contrast to the modular group, we define the traditional flavor group $\mathcal{G}_{\mathrm{fl}}$ by those discrete transformations $g \in \mathcal{G}_{\mathrm{fl}}$ that leave $\tau$ invariant at all points in $\tau$ moduli space, i.e. for all $\tau$

$$
\tau \stackrel{g}{\longmapsto} \tau, \quad \psi \stackrel{g}{\longmapsto} \rho(g) \psi,
$$

where $\rho(g)$ is a (reducible or irreducible) representation of $\mathcal{G}_{\mathrm{fl}}$. Hence, Yukawa couplings $Y(\tau)$ are invariant under transformations from $\mathcal{G}_{\mathrm{fl}}$ for all $\tau$.

As we show next, traditional flavor groups are naturally connected to finite modular groups. To see this, we apply the modular $\mathrm{S}$ transformation eq. (2.3) twice and obtain

$$
\begin{aligned}
& \tau \stackrel{\mathrm{S}}{\longmapsto}-\frac{1}{\tau} \stackrel{\mathrm{S}}{\longmapsto} \tau, \\
& \psi \stackrel{\mathrm{S}}{\longmapsto}(-\tau)^{-k} \rho(\mathrm{S}) \psi \stackrel{\mathrm{S}}{\longmapsto}\left(\frac{1}{\tau}\right)^{-k}(-\tau)^{-k}(\rho(\mathrm{S}))^{2} \psi=(-1)^{-k}(\rho(\mathrm{S}))^{2} \psi .
\end{aligned}
$$

Since the $\tau$ modulus is invariant under $\mathrm{S}^{2}, \mathrm{~S}^{2}$ is by definition part of the traditional flavor group. Moreover, the matter fields $\psi$ transform in general nontrivially, $(-1)^{-k}(\rho(\mathrm{S}))^{2} \neq \mathbb{1}$, 
see also appendix A. Thus, finite modular groups naturally yield traditional flavor groups, and one might wonder how one can in general combine a traditional flavor group consistently with a finite modular group.

To answer this question, we derive a constraint on the extension of a traditional flavor group by a finite modular group, inspired by the discussions in refs. [17, 55-57], where symmetries are extended by $\mathcal{C P}$. In detail, we start with a given traditional flavor group $\mathcal{G}_{\mathrm{ff}}$ and try to extend this group consistently by two generators $\rho(\mathrm{S})$ and $\rho(\mathrm{T})$ of some finite modular group. To do so, let us consider two chains of transformations of the form "modular, flavor, inverse modular",

$$
\begin{aligned}
& \psi \stackrel{\mathrm{S}}{\longmapsto}(-\tau)^{-k} \rho(\mathrm{S}) \psi \stackrel{g}{\longmapsto}(-\tau)^{-k} \rho(\mathrm{S}) \rho(g) \psi \stackrel{\mathrm{S}^{-1}}{\longmapsto} \rho(\mathrm{S}) \rho(g) \rho(\mathrm{S})^{-1} \psi, \\
& \psi \stackrel{\mathrm{T}}{\longmapsto} \quad \rho(\mathrm{T}) \psi \stackrel{g}{\longmapsto} \quad \rho(\mathrm{T}) \rho(g) \psi \stackrel{\mathrm{T}^{-1}}{\longmapsto} \rho(\mathrm{T}) \rho(g) \rho(\mathrm{T})^{-1} \psi,
\end{aligned}
$$

for $g \in \mathcal{G}_{\mathrm{fl}}$. The $\tau$ modulus is invariant under both chains of transformations. Since we do not want to enhance the traditional flavor group $\mathcal{G}_{\mathrm{fl}}$ to a larger traditional flavor group $\mathcal{G}_{\mathrm{fl}}^{\prime}$ by including new generators $\rho(\mathrm{S}) \rho(g) \rho(\mathrm{S})^{-1}$ and $\rho(\mathrm{T}) \rho(g) \rho(\mathrm{T})^{-1}$, we see from eq. (2.9) that

$$
\rho(\mathrm{S}) \rho(g) \rho(\mathrm{S})^{-1} \in \mathcal{G}_{\mathrm{fl}} \quad \text { and } \quad \rho(\mathrm{T}) \rho(g) \rho(\mathrm{T})^{-1} \in \mathcal{G}_{\mathrm{fl}}
$$

must belong to the traditional flavor group $\mathcal{G}_{\text {fl }}$ for all $g \in \mathcal{G}_{\text {fll }}$. In other words, due to eq. (2.10) the traditional flavor group $\mathcal{G}_{\mathrm{fl}}$ must be a normal subgroup of the combined group generated by $\rho(\mathrm{S}), \rho(\mathrm{T})$ and $\rho(g)$, which we call the eclectic flavor group. Moreover, we find

$$
\left\langle\rho(\mathrm{S}) \rho(g) \rho(\mathrm{S})^{-1} \mid g \in \mathcal{G}_{\mathrm{fl}}\right\rangle \cong\left\langle\rho(\mathrm{T}) \rho(g) \rho(\mathrm{T})^{-1} \mid g \in \mathcal{G}_{\mathrm{fl}}\right\rangle \cong \mathcal{G}_{\mathrm{fl}} .
$$

Thus, we can sharpen the constraint (2.10) as

$$
\rho(\mathrm{S}) \rho(g) \rho(\mathrm{S})^{-1}=\rho\left(u_{\mathrm{S}}(g)\right) \quad \text { and } \quad \rho(\mathrm{T}) \rho(g) \rho(\mathrm{T})^{-1}=\rho\left(u_{\mathrm{T}}(g)\right),
$$

where due to eq. (2.11) the maps $u_{\mathrm{S}}$ and $u_{\mathrm{T}}$ are automorphisms of the traditional flavor group $\mathcal{G}_{\mathrm{fl}}$. Since $\rho(\mathrm{S})$ and $\rho(\mathrm{T})$ are assumed to generate a finite modular group, it follows from eq. (2.12) that the automorphisms $u_{\mathrm{S}}$ and $u_{\mathrm{T}}$ have to satisfy the defining relations $(2.6)$, i.e.

$$
\left(u_{\mathrm{S}}\right)^{N_{\mathrm{S}}}=\mathbb{1}, \quad\left(u_{\mathrm{T}}\right)^{N}=\mathbb{1}, \quad\left(u_{\mathrm{S}}\right)^{2} \circ u_{\mathrm{T}}=u_{\mathrm{T}} \circ\left(u_{\mathrm{S}}\right)^{2} \quad \text { and } \quad\left(u_{\mathrm{S}} \circ u_{\mathrm{T}}\right)^{3}=\mathbb{1},
$$

with $N_{\mathrm{S}}=2$ for $\Gamma_{N}$ and $N_{\mathrm{S}}=4$ for $\Gamma_{N}^{\prime}$. Note that the identity $\mathbb{1}$ in eq. (2.13) has to be understood as the trivial automorphism, $\mathbb{1}: g \mapsto g$ for all $g \in \mathcal{G}_{\mathrm{fl}}$, and not as an inner automorphism. As a consequence, the finite modular group defined by eq. (2.13) must be a subgroup of the full automorphism group of the traditional flavor group $\mathcal{G}_{\mathrm{ff}}$.

Now, we can consider two cases. First, if the traditional flavor group commutes with the finite modular group, eq. (2.12) yields

$$
\rho(g)=\rho\left(u_{\mathrm{S}}(g)\right) \quad \text { and } \quad \rho(g)=\rho\left(u_{\mathrm{T}}(g)\right),
$$

for all $g \in \mathcal{G}_{\mathrm{fl}}$. Thus, both modular transformations $\mathrm{S}$ and $\mathrm{T}$ in eq. (2.12) correspond to the trivial automorphism of $\mathcal{G}_{\mathrm{fl}}, u_{\mathrm{S}}=u_{\mathrm{T}}=\mathbb{1}$, and the eclectic flavor group is just given by the direct product extension $\mathcal{G}_{\mathrm{ff}} \times \Gamma_{N}$ or $\mathcal{G}_{\mathrm{ff}} \times \Gamma_{N}^{\prime}$. In this case, the finite modular group can be chosen freely. 
Motivated by the TD approach however, we are interested only in the case where the traditional flavor group does not commute with the finite modular group. In order to satisfy condition (2.12) in this case, the traditional flavor group must have nontrivial automorphisms as candidates for $u_{\mathrm{S}}$ and $u_{\mathrm{T}}$. In general, an automorphism can be inner or outer, where for an outer automorphism $u$ there exists no $h_{u} \in \mathcal{G}_{\mathrm{fl}}$ such that

$$
u(g)=h_{u} g h_{u}^{-1} \quad \text { for all } g \in \mathcal{G}_{\mathrm{fl}} .
$$

Thus, we have to decide whether $u_{\mathrm{S}}$ and $u_{\mathrm{T}}$ are inner or outer automorphisms of the traditional flavor group $\mathcal{G}_{\mathrm{fl}}$.

Let us first assume that $u_{\mathrm{S}}$ and $u_{\mathrm{T}}$ are inner automorphisms of the traditional flavor group $\mathcal{G}_{\mathrm{fl}}$. In this case, the automorphisms $u_{\mathrm{S}}$ and $u_{\mathrm{T}}$ would be defined as

$$
u_{\mathrm{S}}(g)=h_{\mathrm{S}} g h_{\mathrm{S}}^{-1}, \quad u_{\mathrm{T}}(g)=h_{\mathrm{T}} g h_{\mathrm{T}}^{-1},
$$

for some fixed $h_{\mathrm{S}}, h_{\mathrm{T}} \in \mathcal{G}_{\mathrm{fl}}$. Now, assume that $u_{\mathrm{S}}$ and $u_{\mathrm{T}}$ satisfy eq. (2.13) and, hence, generate some finite modular group. Then, the action of the modular generators $\mathrm{S}$ and $\mathrm{T}$ can always be compensated by an element of the flavor group. To be specific, consider the transformations

$$
\psi \stackrel{\mathrm{S}}{\longmapsto}(-\tau)^{-k} \rho(\mathrm{S}) \psi \stackrel{h_{\mathrm{S}}^{-1}}{\longmapsto}(-\tau)^{-k} \mathbb{1} \psi,
$$

and similarly for $\mathrm{T}$, where we used $\rho(\mathrm{S})=\rho\left(h_{\mathrm{S}}\right)$ that follows from eqs. (2.12) and (2.16). Hence, the generators of the finite modular group can be redefined such that the representation of the finite modular group on matter fields $\psi$ is trivial, $\rho(\gamma)=\mathbb{1}$. Since this group would be a trivial extension, we demand in the following that $u_{\mathrm{S}}$ and $u_{\mathrm{T}}$ are outer automorphisms.

Once this requirement is met, $u_{\mathrm{S}}$ and $u_{\mathrm{T}}$ are subject to eqs. (2.12) and (2.13), which impose strong constraints on the possible extensions of the traditional flavor group by a finite modular group.

\subsection{The eclectic extension}

From our previous discussion, one can classify for a given traditional flavor group ${ }^{5} \mathcal{G}_{\mathrm{fl}}$ all nontrivial extensions by finite modular groups as follows:

i) First, one determines the automorphisms of $\mathcal{G}_{\text {fl }}$ and chooses two particular outer automorphisms $u_{\mathrm{S}}$ and $u_{\mathrm{T}}$, whose specific properties shall be motivated and explained in detail in the next section in the context of $\mathcal{C P}$.

ii) Then, one checks whether $u_{\mathrm{S}}$ and $u_{\mathrm{T}}$ satisfy the presentation of a finite modular group as given in eq. (2.13).

iii) Finally, for a given representation $\rho(g)$ of the traditional flavor group, one constructs $\rho(\mathrm{S})$ and $\rho(\mathrm{T})$ explicitly using eq. (2.12) such that $\rho(\mathrm{S})$ and $\rho(\mathrm{T})$ satisfy the presentation eq. (2.6) of the same finite modular group as $u_{\mathrm{S}}$ and $u_{\mathrm{T}}$.

\footnotetext{
${ }^{5}$ See e.g. [58] for an extensive list of possible traditional flavor groups.
} 
The multiplicative closure of the traditional flavor group $\mathcal{G}_{\mathrm{fl}}$ and its compatible finite modular group $\Gamma_{N}$ (or $\Gamma_{N}^{\prime}$ ) is called eclectic flavor group, where a potential extension by a $\mathcal{C P}$-like transformation will be discussed in the next section. Let us stress that the eclectic flavor group is not a direct product of $\mathcal{G}_{\mathrm{fl}}$ and $\Gamma_{N}\left(\right.$ or $\Gamma_{N}^{\prime}$ ) - in other words, $\mathcal{G}_{\mathrm{fl}}$ does not commute with $\Gamma_{N}\left(\right.$ or $\left.\Gamma_{N}^{\prime}\right)$.

\subsection{Combining with $\mathcal{C P}$}

One can combine the modular group $\operatorname{SL}(2, \mathbb{Z})$ with a $\mathcal{C P}$-like transformation by introducing a new generator $K_{*}$, which, on the level of the $2 \times 2$ matrices given in eq. (2.2), can be realized as

$$
\mathrm{K}_{*}=\left(\begin{array}{cc}
1 & 0 \\
0 & -1
\end{array}\right),
$$

such that $\mathrm{SL}(2, \mathbb{Z})$ is enhanced to $\mathrm{GL}(2, \mathbb{Z})[17]$. Under $\mathrm{K}_{*}$, the $\tau$ modulus and the matter fields $\psi(x)$ transform as

$$
\tau \stackrel{\mathrm{K}_{*}}{\longmapsto}-\bar{\tau}, \quad \psi(x) \stackrel{\mathrm{K}_{*}}{\longmapsto} \rho\left(\mathrm{K}_{*}\right) \bar{\psi}\left(x_{P}\right),
$$

see ref. [42] and also $[17,43,59]$. Demanding that the $\mathcal{C P}$-like transformation be of order 2, i.e. $\left(\mathrm{K}_{*}\right)^{2}=\mathbb{1}$, implies $^{6}$

$$
\psi(x) \stackrel{\mathrm{K}_{*}}{\longmapsto} \rho\left(\mathrm{K}_{*}\right) \bar{\psi}\left(x_{P}\right) \stackrel{\mathrm{K}_{*}}{\longmapsto} \rho\left(\mathrm{K}_{*}\right) \rho\left(\mathrm{K}_{*}\right)^{*} \psi(x) \stackrel{!}{=} \psi(x)
$$

and therefore

$$
\rho\left(\mathrm{K}_{*}\right) \rho\left(\mathrm{K}_{*}\right)^{*}=\mathbb{1} \quad \Leftrightarrow \quad \rho\left(\mathrm{K}_{*}\right)^{*}=\rho\left(\mathrm{K}_{*}\right)^{-1} .
$$

In general, the additional generator $\rho\left(\mathrm{K}_{*}\right)$ of $\mathcal{C P}$ does not commute with $\Gamma_{N}\left(\right.$ or $\left.\Gamma_{N}^{\prime}\right)$. To see this, let us first consider the chain of transformations

$$
\begin{aligned}
\psi(x) \stackrel{\mathrm{K}_{*}}{\longmapsto} & \rho\left(\mathrm{K}_{*}\right) \bar{\psi}\left(x_{P}\right) \stackrel{\mathrm{S}}{\longmapsto}(-\bar{\tau})^{-k} \rho\left(\mathrm{K}_{*}\right) \rho(\mathrm{S})^{*} \bar{\psi}\left(x_{P}\right) \\
& \stackrel{\mathrm{K}_{*}}{\longmapsto}(+\tau)^{-k} \rho\left(\mathrm{K}_{*}\right) \rho(\mathrm{S})^{*} \rho\left(\mathrm{K}_{*}\right)^{-1} \psi(x) \\
& \stackrel{!}{=}(+\tau)^{-k} \rho(\gamma) \psi(x)
\end{aligned}
$$

for some modular transformation $\gamma \in \mathrm{SL}(2, \mathbb{Z})$ that we determine next. Under the chain of transformations eq. (2.22) the $\tau$ modulus transforms as $\tau \mapsto-1 / \tau$. Thus, $\gamma=\mathrm{S}_{\text {or }} \mathrm{S}^{-1}$ from eq. (2.2). Eq. (2.22c) implies that the solution is $\gamma=\mathrm{S}^{-1}$. In summary, we have found that $\mathrm{K}_{*} \mathrm{SK}_{*}=\mathrm{S}^{-1}$ on the level of $\mathrm{GL}(2, \mathbb{Z})$. Consequently, the finite modular group has to be extended by $\rho\left(\mathrm{K}_{*}\right)$ satisfying

$$
\rho\left(\mathrm{K}_{*}\right) \rho(\mathrm{S})^{*} \rho\left(\mathrm{K}_{*}\right)^{-1}=\rho(\mathrm{S})^{-1} .
$$

\footnotetext{
${ }^{6}$ Note that it is in principle possible that $\mathcal{C P}$ is not of order 2 [55-57]. However, in this case $\mathrm{K}_{*}^{2}$ acts as $\tau \mapsto \tau$ and $\psi(x) \mapsto \rho\left(K_{*}\right) \rho\left(K_{*}\right)^{*} \psi(x)$, which implies that $\rho\left(K_{*}\right) \rho\left(K_{*}\right)^{*}$ is from the traditional flavor group. As we do not want to extend the traditional flavor group by further traditional flavor transformations (like $\left.\rho\left(K_{*}\right) \rho\left(K_{*}\right)^{*}\right)$, we focus on the case $\left(K_{*}\right)^{2}=\mathbb{1}$.
} 
By repeating these steps for $\mathrm{T}$, we find $\mathrm{K}_{*} \mathrm{~T} \mathrm{~K}_{*}=\mathrm{T}^{-1}$ and, as the final result, that the conditions (2.6) get extended by

$$
\begin{aligned}
\rho\left(\mathrm{K}_{*}\right)^{*} & =\rho\left(\mathrm{K}_{*}\right)^{-1}, \\
\rho\left(\mathrm{K}_{*}\right) \rho(\mathrm{S})^{*} \rho\left(\mathrm{K}_{*}\right)^{-1} & =\rho(\mathrm{S})^{-1}, \\
\rho\left(\mathrm{K}_{*}\right) \rho(\mathrm{T})^{*} \rho\left(\mathrm{K}_{*}\right)^{-1} & =\rho(\mathrm{T})^{-1} .
\end{aligned}
$$

This enhances the finite modular group $\Gamma_{N}$ to $\Gamma_{N}^{*}$ (and enhances $\Gamma_{N}^{\prime}$ to $\Gamma_{N}^{*}$ ), defined as

$$
\begin{gathered}
\Gamma_{N}^{*}=\left\langle\mathrm{S}, \mathrm{T}, \mathrm{K}_{*}\right| \mathrm{S}^{2}=\mathbb{1},(\mathrm{ST})^{3}=\mathbb{1}, \mathrm{T}^{N}=\mathbb{1}, \\
\left.\mathrm{K}_{*}^{2}=\mathbb{1}, \mathrm{K}_{*} \mathrm{SK}_{*}=\mathrm{S}^{-1}, \mathrm{~K}_{*} \mathrm{~T} \mathrm{~K}_{*}=\mathrm{T}^{-1}\right\rangle, \\
\Gamma_{N}^{*}=\left\langle\mathrm{S}, \mathrm{T}, \mathrm{K}_{*}\right| \mathrm{S}^{4}=\mathbb{1},(\mathrm{ST})^{3}=\mathbb{1}, \mathrm{T}^{N}=\mathbb{1}, \mathrm{S}^{2} \mathrm{~T}=\mathrm{TS}^{2}, \\
\left.\mathrm{~K}_{*}^{2}=\mathbb{1}, \mathrm{K}_{*} \mathrm{SK}_{*}=\mathrm{S}^{-1}, \mathrm{~K}_{*} \mathrm{~T} \mathrm{~K}_{*}=\mathrm{T}^{-1}\right\rangle .
\end{gathered}
$$

All finite modular groups with a $\mathcal{C P}$-like extension and $N=2,3,4,5$ are listed in table 1.

Next, we discuss how $\Gamma_{N}^{*}$ can be made compatible with the traditional flavor group $\mathcal{G}_{\text {fl }}$, cf. [55-57]. With the additional element $\mathrm{K}_{*}$, our previous discussion proceeds directly. First, eq. (2.9) includes now also the chain of transformations

$$
\psi(x) \stackrel{\mathrm{K}_{*}}{\longmapsto} \rho\left(\mathrm{K}_{*}\right) \bar{\psi}\left(x_{P}\right) \stackrel{g}{\longmapsto} \rho\left(\mathrm{K}_{*}\right) \rho(g)^{*} \bar{\psi}\left(x_{P}\right) \stackrel{\mathrm{K}_{*}^{-1}}{\longmapsto} \rho\left(\mathrm{K}_{*}\right) \rho(g)^{*} \rho\left(\mathrm{K}_{*}\right)^{-1} \psi(x),
$$

which implies $\rho\left(\mathrm{K}_{*}\right) \rho(g)^{*} \rho\left(\mathrm{K}_{*}\right)^{-1} \in \mathcal{G}_{\mathrm{fl}}$ when we prevent $\mathcal{G}_{\mathrm{fl}}$ from being trivially extended by the elements $\rho\left(\mathrm{K}_{*}\right) \rho(g)^{*} \rho\left(\mathrm{K}_{*}\right)^{-1}$. It then follows that there exists an automorphism $u_{\mathrm{K}_{*}}$, such that

$$
\rho\left(\mathrm{K}_{*}\right) \rho(g)^{*} \rho\left(\mathrm{K}_{*}\right)^{-1}=\rho\left(u_{\mathrm{K}_{*}}(g)\right) .
$$

As shown in ref. [57], eq. (2.27) is satisfied by a class-inverting outer automorphism of the traditional flavor group $\mathcal{G}_{\text {ffl }}$. However, one can also have a situation in which not all irreducible representations of $\mathcal{G}_{\mathrm{fl}}$ appear in the theory and there exists an automorphism $u_{\mathrm{K}_{*}}$ satisfying eq. (2.27) only for the representation(s) $\rho$ present in the spectrum. Such an automorphism could then be seen as a $\rho$-restricted class-inverting automorphism. ${ }^{7}$ Now, since this type of outer automorphisms necessarily doubles the dimensions of (some of) the representations and because in the TD approach they give rise to $\mathcal{C P}$-like transformations [42, 43], we reserve these $\rho$-restricted class-inverting automorphisms of $\mathcal{G}_{\mathrm{fl}}$ exclusively for $\mathcal{C P}$.

Consequently, the automorphisms $u_{\mathrm{K}_{*}}, u_{\mathrm{S}}$ and $u_{\mathrm{T}}$ of the traditional flavor group $\mathcal{G}_{\mathrm{fl}}$ have to satisfy (in addition to eq. (2.13)) the conditions

$$
\left(u_{\mathrm{K}_{*}}\right)^{2}=\mathbb{1}, \quad u_{\mathrm{K}_{*}} \circ u_{\mathrm{S}} \circ u_{\mathrm{K}_{*}}=u_{\mathrm{S}}^{-1}, \quad u_{\mathrm{K}_{*}} \circ u_{\mathrm{T}} \circ u_{\mathrm{K}_{*}}=u_{\mathrm{T}}^{-1} .
$$

In more detail, by applying the definitions of the automorphisms $u_{\mathrm{S}}$ and $u_{\mathrm{K}_{*}}$ in eqs. (2.12) and (2.27), the second relation is obtained as follows:

$$
\begin{aligned}
\rho\left(u_{\mathrm{K}_{*}} \circ u_{\mathrm{S}} \circ u_{\mathrm{K}_{*}}(g)\right) & =\rho\left(\mathrm{K}_{*}\right) \rho\left(u_{\mathrm{S}} \circ u_{\mathrm{K}_{*}}(g)\right)^{*} \rho\left(\mathrm{K}_{*}\right)^{-1} \\
& =\rho\left(\mathrm{K}_{*}\right) \rho(\mathrm{S})^{*} \rho\left(u_{\mathrm{K}_{*}}(g)\right)^{*} \rho(\mathrm{S})^{*-1} \rho\left(\mathrm{K}_{*}\right)^{-1} \\
& =\rho\left(\mathrm{K}_{*}\right) \rho(\mathrm{S})^{*} \rho\left(\mathrm{K}_{*}\right)^{-1} \rho(g) \rho\left(\mathrm{K}_{*}\right) \rho(\mathrm{S})^{*-1} \rho\left(\mathrm{K}_{*}\right)^{-1} \\
& =\rho(\mathrm{S})^{-1} \rho(g) \rho(\mathrm{S})=\rho\left(u_{\mathrm{S}}^{-1}(g)\right) .
\end{aligned}
$$

\footnotetext{
${ }^{7}$ We thank Andreas Trautner for useful comments on the properties of class-inverting automorphisms.
} 
As before, for each traditional flavor group $\mathcal{G}_{\mathrm{fl}}$, it is possible to classify all finite modular groups $\Gamma_{N}^{*}$ (and $\Gamma_{N}^{* *}$ ), endowed with a $\mathcal{C P}$-like transformation, that are compatible with $\mathcal{G}_{\mathrm{ff}}$. One must first create all automorphisms of $\mathcal{G}_{\mathrm{fl}}$ and choose outer automorphisms $u_{\mathrm{S}}, u_{\mathrm{T}}$ and $u_{\mathrm{K}_{*}}$ satisfying the presentation of an enhanced finite modular group given by eqs. (2.13) and (2.28). Finally, one must explicitly find the representations $\rho(\mathrm{S}), \rho(\mathrm{T})$ and $\rho\left(\mathrm{K}_{*}\right)$ that fulfill eqs. (2.12), (2.24) and (2.27).

Let us make a remark on the non-Abelian structure of the $\mathcal{C P}$-like extension of finite modular groups. Despite the fact that eq. (2.25a) indicates that, for $N>2$, the generator $\mathrm{K}_{*}$ of $\mathcal{C P}$ does not commute with $\mathrm{S}$ and $\mathrm{T}$, the $\mathcal{C P}$-like extension of $\Gamma_{N}$ becomes $\Gamma_{N}^{*}=\Gamma_{N} \times \mathbb{Z}_{2}$ for several finite modular groups. In detail, the $\mathbb{Z}_{2}$ factors in the $\Gamma_{N}^{*}$ finite modular groups $S_{3} \times \mathbb{Z}_{2}, S_{4} \times \mathbb{Z}_{2}$ and $A_{5} \times \mathbb{Z}_{2}$ are generated by $\mathrm{K}_{*},\left(\mathrm{~T}^{2} \mathrm{~S}\right)^{2} \mathrm{~K}_{*}$ and $\mathrm{S}\left(\mathrm{T}^{2} \mathrm{ST}\right)^{2} \mathrm{TK}_{*}$, respectively, see table 1 .

\section{Local flavor unification}

We are considering a setting, where modular and traditional flavor symmetries do not commute. This gives rise to the picture of "Local Flavor Unification" [42, 43]: at so-called self-dual points or lines in moduli space $\langle\tau\rangle$ the finite modular symmetry is broken spontaneously to those subgroups that leave $\langle\tau\rangle$ invariant. In contrast, the traditional flavor symmetry, by definition, leaves the modulus $\tau$ invariant and, hence, remains unbroken everywhere in moduli space. As modular and traditional flavor symmetries do not commute, the unbroken modular transformations yield nontrivial enhancements of the traditional flavor symmetry to the so-called unified flavor symmetries at the self-dual points in moduli space.

Let us begin the discussion with the finite modular group $\Gamma_{N}$ or $\Gamma_{N}^{\prime}$, i.e. without taking $\mathcal{C P}$-like transformations into account. Then, if the modulus is stabilized at $\langle\tau\rangle=\mathrm{i}$, the following modular transformation remains unbroken

$$
\langle\tau\rangle \stackrel{\mathrm{S}}{\longmapsto}-\frac{1}{\langle\tau\rangle}=\langle\tau\rangle \quad \text { at } \quad\langle\tau\rangle=\mathrm{i},
$$

see also the related discussion in ref. [34]. At this point in moduli space, matter fields transform as

$$
\psi \stackrel{g}{\longmapsto} \rho(g) \psi \quad \text { for } \quad g \in \mathcal{G}_{\mathrm{fl}} \quad \text { and } \quad \psi \stackrel{g}{\longmapsto}(-\mathrm{i})^{-k} \rho(g) \psi \text { for } \quad g=\mathrm{S} .
$$

Thus, at $\langle\tau\rangle=\mathrm{i}$ the traditional flavor symmetry $\mathcal{G}_{\mathrm{fl}}$ is enhanced by the generator S. Since $\rho(\mathrm{S})$ is either of order $N_{\mathrm{S}}=2$ or $N_{\mathrm{S}}=4$, see eq. (2.6), the unified flavor symmetry at $\langle\tau\rangle=\mathrm{i}$ is a nontrivial extension of $\mathcal{G}_{\mathrm{fl}}$ by $\mathbb{Z}_{N_{\mathrm{S}}}[42,43]$, or even of higher order depending on the modular weight $k \in \mathbb{Q}$.

If the modulus is stabilized at $\langle\tau\rangle=\exp (2 \pi \mathrm{i} / 3)$, we find the following unbroken modular transformation

$$
\langle\tau\rangle \stackrel{\mathrm{ST}}{\longmapsto}-\frac{1}{\langle\tau\rangle+1}=\langle\tau\rangle \quad \text { at } \quad\langle\tau\rangle=\exp (2 \pi \mathrm{i} / 3) \quad \text { for } \quad \mathrm{ST}=\left(\begin{array}{cc}
0 & 1 \\
-1 & -1
\end{array}\right)
$$


Thus, at $\langle\tau\rangle=\exp (2 \pi \mathrm{i} / 3)$ the traditional flavor symmetry is enhanced by $\mathrm{S} \mathrm{T}$ and matter fields transform as

$$
\psi \stackrel{g}{\longmapsto} \rho(g) \psi \quad \text { for } \quad g \in \mathcal{G}_{\mathrm{fl}} \quad \text { and } \quad \psi \stackrel{g}{\longmapsto} \exp (2 \pi \mathrm{i} k / 3) \rho(g) \psi \text { for } g=\mathrm{ST}
$$

under the unified flavor symmetry at $\langle\tau\rangle=\exp (2 \pi \mathrm{i} / 3)$. Note that $\rho(\mathrm{ST})=\rho(\mathrm{S}) \rho(\mathrm{T})$ is of order 3 , see eq. (2.6). Thus, the unified flavor symmetry at $\langle\tau\rangle=\exp (2 \pi \mathrm{i} / 3)$ is a nontrivial product of $\mathcal{G}_{\mathrm{fl}}$ and a $\mathbb{Z}_{3}$ generated by $\mathrm{ST}$ (if $k \in \mathbb{Z}$ ).

If one includes the $\mathcal{C P}$-like transformation, additional modular transformations can remain unbroken. For example, at vertical lines in moduli space given by $\langle\tau\rangle=n_{B} / 2+\mathrm{i} \sqrt{3} r / 2$ with $n_{B} \in \mathbb{Z}$ and $r \in \mathbb{R}$ we find that $\mathrm{T}^{n_{B}} \mathrm{~K}_{*}$ remains unbroken [42, 43], i.e.

$$
\langle\tau\rangle \stackrel{\mathrm{T}_{B}^{n_{B} \mathrm{~K}_{*}} \longmapsto}{\longmapsto} n_{B}-\overline{\langle\tau\rangle}=\langle\tau\rangle \quad \text { at } \quad\langle\tau\rangle=\frac{n_{B}}{2}+\mathrm{i} \frac{\sqrt{3}}{2} r \quad \text { for } \quad \mathrm{T}^{n_{B}} \mathrm{~K}_{*}=\left(\begin{array}{cc}
1 & -n_{B} \\
0 & -1
\end{array}\right) .
$$

In particular, for $n_{B}=0$, i.e. on the vertical line $\langle\tau\rangle=\mathrm{i} \sqrt{3} r / 2$ we obtain an unbroken modular transformation $\mathrm{K}_{*}$, while at $\langle\tau\rangle=$ i eq. (3.1) yields an unbroken $\mathrm{S}$ transformation. Moreover, if one moves away from $\langle\tau\rangle=\mathrm{i}$ but stays on the circle $\langle\tau\rangle=\exp (\mathrm{i} \alpha)$, only the combined transformation $\mathrm{K}_{*} \mathrm{~S}$ remains unbroken

$$
\langle\tau\rangle \stackrel{\mathrm{K}_{*} \mathrm{~S}}{\longmapsto} \frac{1}{\overline{\langle\tau\rangle}}=\langle\tau\rangle \quad \text { at } \quad\langle\tau\rangle=\exp (\mathrm{i} \alpha) \quad \text { for } \quad \mathrm{K}_{*} \mathrm{~S}=\left(\begin{array}{ll}
0 & 1 \\
1 & 0
\end{array}\right) .
$$

At these lines in moduli space, a $\mathcal{C P}$-like transformation is unbroken. However, it is easy to break $\mathcal{C P}$ spontaneously by moving $\langle\tau\rangle$ away from these symmetry enhanced lines in moduli space.

\section{Example: $\Delta(54)$ and modular symmetries}

As an example, let us discuss the possible extensions of the traditional flavor symmetry $\Delta(54)[60-63] . \Delta(54)$ is a non-Abelian group of order 54 that has three-dimensional representations suitable for the three generations of quarks and leptons. In detail, $\Delta(54)$ can be generated by three generators $\mathrm{A}, \mathrm{B}$, and $\mathrm{C}$ subject to the presentation

$$
\Delta(54)=\left\langle\mathrm{A}, \mathrm{B}, \mathrm{C} \mid \mathrm{A}^{3}=\mathrm{B}^{3}=\mathrm{C}^{2}=(\mathrm{AC})^{2}=(\mathrm{BC})^{2}=(\mathrm{AB})^{3}=\left(\mathrm{AB}^{2}\right)^{3}=\mathbb{1}\right\rangle .
$$

A three-dimensional representation of $\Delta(54)$ is given by

$$
\rho(\mathrm{A})=\left(\begin{array}{lll}
0 & 1 & 0 \\
0 & 0 & 1 \\
1 & 0 & 0
\end{array}\right), \quad \rho(\mathrm{B})=\left(\begin{array}{ccc}
1 & 0 & 0 \\
0 & \omega & 0 \\
0 & 0 & \omega^{2}
\end{array}\right), \quad \rho(\mathrm{C})=\left(\begin{array}{ccc}
-1 & 0 & 0 \\
0 & 0 & -1 \\
0 & -1 & 0
\end{array}\right),
$$

where $\omega:=\exp 2 \pi \mathrm{i} / 3$. Since the group of outer automorphisms 8

$$
\operatorname{Out}(\Delta(54)) \cong \operatorname{Aut}(\Delta(54)) / \operatorname{Inn}(\Delta(54)) \cong S_{4}
$$

\footnotetext{
${ }^{8}$ Using GAP [50] we obtain Aut $(\Delta(54)) \cong[432,734]$ and $\operatorname{Inn}(\Delta(54)) \cong[18,4]$ being the generalized dihedral group.
} 
is nontrivial, there are outer automorphisms that might in principle lead to eclectic extensions of the traditional flavor symmetry $\Delta(54)$. It turns out that $\Delta(54)$ can be extended only in two ways: either by the finite modular group $\Gamma_{3}^{\prime} \cong T^{\prime}$ in the case without $\mathcal{C P}$ or by $\Gamma_{3}^{\prime *} \cong \mathrm{SL}(2,3)$ in the case with $\mathcal{C P}$. The details are given in the following:

Finite modular symmetry $\boldsymbol{\Gamma}_{\mathbf{3}}^{\prime}$. We can choose the outer automorphisms of $\Delta(54)$

$$
\begin{array}{ll}
u_{\mathrm{S}}(\mathrm{A})=\mathrm{B}^{2}, & u_{\mathrm{T}}(\mathrm{A})=\mathrm{B} A \mathrm{~B}, \\
u_{\mathrm{S}}(\mathrm{B})=\mathrm{A}, & u_{\mathrm{T}}(\mathrm{B})=\mathrm{B}, \\
u_{\mathrm{S}}(\mathrm{C})=\mathrm{C}, & u_{\mathrm{T}}(\mathrm{C})=\mathrm{C} .
\end{array}
$$

Using the presentation eq. (2.6), one can verify that $u_{\mathrm{S}}$ and $u_{\mathrm{T}}$ generate the finite modular group $\Gamma_{3}^{\prime} \cong T^{\prime} \cong \mathrm{SL}(2,3)$. For the three-dimensional representation eq. (4.2) of $\Delta(54)$ these outer automorphisms can be written by conjugation with ${ }^{9}$

$$
\rho(\mathrm{S})=-\frac{\mathrm{i}}{\sqrt{3}}\left(\begin{array}{ccc}
1 & 1 & 1 \\
1 & \omega & \omega^{2} \\
1 & \omega^{2} & \omega
\end{array}\right) \quad \text { and } \quad \rho(\mathrm{T})=\left(\begin{array}{ccc}
\omega & 0 & 0 \\
0 & 1 & 0 \\
0 & 0 & 1
\end{array}\right)
$$

see eq. (2.12), where possible phases have been fixed by demanding that $\rho(\mathrm{S})$ and $\rho(\mathrm{T})$ generate the same finite modular group $\Gamma_{3}^{\prime} \cong T^{\prime} \cong \mathrm{SL}(2,3)$ as $u_{\mathrm{S}}$ and $u_{\mathrm{T}}$. By inspecting the character table of $T^{\prime}$, it is easy to see that eq. (4.5) corresponds to the $\mathbf{1} \oplus \mathbf{2}^{\prime \prime}$ irreducible representations of $T^{\prime}$. Furthermore, we note that $(\rho(\mathrm{S}))^{2}=\rho(\mathrm{C})$ and the three-dimensional reducible representation eq. (4.5) of $T^{\prime}$ is an irreducible representation of the eclectic flavor group generated by $\rho(\mathrm{A}), \rho(\mathrm{B}), \rho(\mathrm{S})$ and $\rho(\mathrm{T})$, being

$$
\Omega(1) \cong[648,533],
$$

see ref. [64] for the definition of $\Omega(1)$ and refs. [65, 66] for flavor model building based on this symmetry group. Following the discussion in section 3, the eclectic flavor group $\Omega(1)$ is broken spontaneously to various unified flavor subgroups at different points in $\tau$ moduli space:

$$
\begin{array}{rll}
\text { generic point : } & \Omega(1) \rightarrow \Delta(54) & \cong[54,8] \\
\langle\tau\rangle=\mathrm{i}: \Omega(1) \rightarrow \Sigma(36 \times 3) & \cong[108,15] \\
\langle\tau\rangle=\exp (2 \pi \mathrm{i} / 3): \Omega(1) \rightarrow \tilde{Y}(0) & \cong[162,10]
\end{array}
$$

where the flavor groups are defined as follows:

i) $\Delta(54)$ is generated by $\rho(\mathrm{A}), \rho(\mathrm{B})$ and $\rho(\mathrm{C})$,

ii) $\Sigma(36 \times 3) \cong[108,15]$ is generated by $\rho(\mathrm{A}), \rho(\mathrm{B})$ and $\rho(\mathrm{S})$ (see ref. [67]), and, finally,

iii) $\tilde{Y}(0) \cong[162,10]$ is generated by $\rho(\mathrm{A}), \rho(\mathrm{B}), \rho(\mathrm{C})$ and $\rho(\mathrm{S} \mathrm{T})$ (see ref. [64]).

As a remark, all of these groups have three-dimensional irreducible representations.

\footnotetext{
${ }^{9}$ Note the change of convention compared to ref. [43].
} 
$\mathcal{C P}$-like modular extension. Following the discussion of section 2.2 , it is possible to extend the identified modular symmetry $T^{\prime}$ with a $\mathcal{C P}$-like transformation $\mathrm{K}_{*}$ based on the $\Delta(54)$ outer automorphism

$$
u_{\mathrm{K}_{*}}(\mathrm{~A})=\mathrm{A}, \quad u_{\mathrm{K}_{*}}(\mathrm{~B})=\mathrm{B}^{2}, \quad u_{\mathrm{K}_{*}}(\mathrm{C})=\mathrm{C}
$$

that satisfies the conditions (2.28) with $u_{\mathrm{S}}$ and $u_{\mathrm{T}}$ given by eqs. (4.4). In this case, one can see from eq. (2.27) that the representation $\rho\left(\mathrm{K}_{*}\right)$ of eq. (2.19) relating the field $\psi(x)$ in the $\mathbf{3}$ representation of $\Delta(54)$ with its conjugate $\bar{\psi}\left(x_{P}\right)$ in the $\overline{\mathbf{3}}$ representation is given by

$$
\rho\left(\mathrm{K}_{*}\right)=\mathbb{1}_{3 \times 3} .
$$

Since $\mathrm{K}_{*}$ relates $\psi(x)$ and $\bar{\psi}\left(x_{P}\right)$, it is more convenient to rewrite $\mathrm{S}, \mathrm{T}$ and $\mathrm{K}_{*}$ in the $\mathbf{3} \oplus \overline{\mathbf{3}}$ representation as

$$
\rho_{\mathbf{6}}(\mathrm{S})=\left(\begin{array}{cc}
\rho(\mathrm{S}) & 0 \\
0 & \rho(\mathrm{S})^{*}
\end{array}\right), \quad \rho_{\mathbf{6}}(\mathrm{T})=\left(\begin{array}{cc}
\rho(\mathrm{T}) & 0 \\
0 & \rho(\mathrm{T})^{*}
\end{array}\right), \quad \rho_{\mathbf{6}}\left(\mathrm{K}_{*}\right)=\left(\begin{array}{cc}
0 & \rho\left(\mathrm{K}_{*}\right) \\
\rho\left(\mathrm{K}_{*}\right)^{*} & 0
\end{array}\right)
$$

where $\rho(\mathrm{S})$ and $\rho(\mathrm{T})$ are given by eq. (4.5) and $\rho\left(\mathrm{K}_{*}\right)$ by eq. (4.9). One can easily verify that $\rho_{\mathbf{6}}(\mathrm{S}), \rho_{\mathbf{6}}(\mathrm{T})$ and $\rho_{\mathbf{6}}\left(\mathrm{K}_{*}\right)$ generate the finite modular group $\Gamma_{3}^{*} \cong \mathrm{GL}(2,3)$ and the resulting eclectic flavor group is [1296, 2891].

In extra-dimensional models, the traditional flavor symmetry $\Delta(54)$ can originate from strings on a $\mathbb{T}^{2} / \mathbb{Z}_{3}$ orbifold [48,68]. As shown in refs. [42, 43], $\Delta(54)$ is accompanied nontrivially in this setting by a $T^{\prime}$ or a $\operatorname{GL}(2,3)$ modular symmetry, depending on whether the $\mathcal{C P}$-like transformation $\mathrm{K}_{*}$ is taken into account, in full agreement with our discussion here, see also ref. [69].

\section{Results}

We have selected a representative set of traditional flavor symmetries that have been used in model building. These include

$$
\begin{aligned}
& S_{3}, Q_{8}, \mathbb{Z}_{3} \times \mathbb{Z}_{3}, A_{4}, S_{3} \times \mathbb{Z}_{2}, T_{7}, S_{4}, T^{\prime}, \Delta(27), \mathbb{Z}_{9} \rtimes \mathbb{Z}_{3} \cong[27,4], \operatorname{SL}(2,4), \\
& \Delta(54), A_{5}, \Sigma(36 \times 3) \cong[108,15], \Sigma(168) \cong[168,42] \text { and } \Sigma(72 \times 3) \cong[216,88] .
\end{aligned}
$$

This list is not exhaustive, but covers the most promising traditional flavor symmetries considered so far. More examples could be added and analyzed upon request. We then classify for each group all nontrivial extensions by finite modular symmetries that fulfill the restrictions discussed in section 2. This then allows the identification of allowed eclectic flavor groups that could be obtained in a bottom-up procedure. Our results are listed in table 2 . Surprisingly it turns out that many prominent traditional flavor symmetries, such as $S_{3}$, $D_{12} \cong S_{3} \times \mathbb{Z}_{2}, T_{7}, S_{4}, \mathbb{Z}_{9} \rtimes \mathbb{Z}_{3}, \mathrm{SL}(2,4)$ and $A_{5}$, do not allow for any nontrivial modular extension and thus are not included in table 2. Among the groups we have studied, only

$$
Q_{8}, \mathbb{Z}_{3} \times \mathbb{Z}_{3}, A_{4}, T^{\prime}, \Delta(27) \text { and } \Delta(54)
$$




\begin{tabular}{|c|c|c|c|c|c|}
\hline $\begin{array}{c}\text { flavor group } \\
\mathcal{G}_{\mathrm{fl}}\end{array}$ & $\begin{array}{l}\text { GAP } \\
\text { ID }\end{array}$ & $\operatorname{Aut}\left(\mathcal{G}_{\mathrm{fl}}\right)$ & \multicolumn{2}{|c|}{$\begin{array}{c}\text { finite modular } \\
\text { groups }\end{array}$} & $\begin{array}{c}\text { eclectic flavor } \\
\text { group }\end{array}$ \\
\hline \multirow[t]{2}{*}{$Q_{8}$} & \multirow[t]{2}{*}[8,4]{} & \multirow[t]{2}{*}{$S_{4}$} & without $\mathcal{C P}$ & $S_{3}$ & $\operatorname{GL}(2,3)$ \\
\hline & & & with $\mathcal{C P}$ & - & - \\
\hline \multirow[t]{2}{*}{$\mathbb{Z}_{3} \times \mathbb{Z}_{3}$} & \multirow[t]{2}{*}[9,2]{} & \multirow[t]{2}{*}{$\operatorname{GL}(2,3)$} & without $\mathcal{C P}$ & $S_{3}$ & $\Delta(54)$ \\
\hline & & & with $\mathcal{C P}$ & $S_{3} \times \mathbb{Z}_{2}$ & {$[108,17]$} \\
\hline \multirow[t]{3}{*}{$A_{4}$} & \multirow[t]{3}{*}[12,3]{} & \multirow[t]{3}{*}{$S_{4}$} & without $\mathcal{C P}$ & $S_{3}$ & $S_{4}$ \\
\hline & & & & $S_{4}$ & $S_{4}$ \\
\hline & & & with $\mathcal{C P}$ & - & - \\
\hline \multirow[t]{2}{*}{$T^{\prime}$} & \multirow[t]{2}{*}[24,3]{} & \multirow[t]{2}{*}{$S_{4}$} & without $\mathcal{C P}$ & $S_{3}$ & $\mathrm{GL}(2,3)$ \\
\hline & & & with $\mathcal{C P}$ & - & - \\
\hline \multirow[t]{4}{*}{$\Delta(27)$} & \multirow[t]{4}{*}[27,3]{} & \multirow[t]{4}{*}[432,734]{} & without $\mathcal{C P}$ & $S_{3}$ & $\Delta(54)$ \\
\hline & & & & $T^{\prime}$ & $\Omega(1)$ \\
\hline & & & with $\mathcal{C P}$ & $S_{3} \times \mathbb{Z}_{2}$ & {$[108,17]$} \\
\hline & & & & $\operatorname{GL}(2,3)$ & {$[1296,2891]$} \\
\hline \multirow[t]{2}{*}{$\Delta(54)$} & \multirow[t]{2}{*}[54,8]{} & \multirow[t]{2}{*}[432,734]{} & without $\mathcal{C P}$ & $T^{\prime}$ & $\Omega(1)$ \\
\hline & & & with $\mathcal{C P}$ & $\mathrm{GL}(2,3)$ & {$[1296,2891]$} \\
\hline
\end{tabular}

Table 2. Examples of traditional flavor groups, their extensions by finite modular groups and the resulting eclectic flavor groups. For details, see appendix B. Aut $\left(\mathcal{G}_{\mathrm{ff}}\right)$ denotes the group of automorphisms of the traditional flavor group $\mathcal{G}_{\mathrm{ff}}$.

allow for eclectic extensions and local flavor unification. Furthermore, among those, only the traditional flavor groups

$$
\mathbb{Z}_{3} \times \mathbb{Z}_{3}, \Delta(27) \text { and } \Delta(54)
$$

admit a $\mathcal{C P}$-like transformation in the eclectic flavor group.

This is a quite restrictive situation. A nontrivial extension of the traditional flavor group (as required from the top-down argumentation) is limited to just a few specific cases. Flavor model building should thus be based on very few examples with an eclectic flavor group that includes a nontrivial finite modular group. Since our list of traditional flavor groups is not exhaustive, it remains to be seen in future work whether more nontrivial cases can be found. Traditional flavor groups with a sizable group of outer automorphism are particularly suited for an eclectic extension.

\section{Conclusions}

In the present paper we made an effort to match the bottom-up (BU) and top-down (TD) approaches of flavor models based on finite discrete modular symmetries. Up to now, the BU-approach considered finite modular groups $\Gamma_{N}$, where some of the quarks and leptons 
transform as (irreducible) triplet or nontrivial singlet representations of $\Gamma_{N}$. This led to an excellent description of the flavor structure of the lepton sector. Efforts towards an ultraviolet completion (TD approach) were based on string theory. Up to now only few explicit TD models have been constructed. The analysis within string theory, however, leads to a general qualitative picture with the clear message that finite modular symmetries do not appear in isolated form, but are accompanied by a traditional (non-modular) flavor group. This then leads us to the concept of "Eclectic Flavor Groups" as a nontrivial product of traditional flavor symmetry and finite modular symmetry.

Given this observation, we might now reconsider the BU-approach and classify candidates for eclectic flavor groups from bottom-up. Surprisingly, the number of these candidates turns out to be very small. Only a few examples are known (see table 2). This is the main result of the present paper. The fact that we cannot disentangle traditional flavor symmetries and modular symmetries is consistent with the picture of "Local Flavor Unification", where we find an enhanced symmetry at specific regions in moduli space. This would naturally allow different flavor structures for quarks and leptons, where quarks (leptons) are predominantly described by traditional (modular) flavor groups.

It should be stressed that the concept of eclectic flavor groups is more predictive than the consideration of modular symmetries alone. Terms allowed by the modular group might be forbidden by the selection rules of the traditional flavor group. In the $\Delta(54)$ example discussed in section 4 , four independent trilinear superpotential couplings (of $\mathbf{2}^{\prime \prime} \oplus \mathbf{1}$ representations of $T^{\prime}$ ) allowed by the $T^{\prime}$ modular symmetry are reduced to a single one due to the presence of $\Delta(54)$ [70]. The enhanced restrictions from eclectic flavor groups are especially relevant for the form of the Kähler potential. In ref. [33] it was pointed out that general terms in the Kähler potential reduce the predictivity of models based on finite modular symmetries. This problem could be solved within the eclectic flavor picture with more restrictions on the Kähler potential due to the nontrivial combination of finite modular groups and traditional flavor groups.

\section{Acknowledgments}

We are thankful to Andreas Trautner for useful observations. H.P.N. is supported by the Excellence Cluster ORIGINS, funded by the Deutsche Forschungsgemeinschaft (DFG, German Research Foundation) under Germany's Excellence Strategy — EXC-2094 390783311. The work of S.R.-S. was partly supported by DGAPA-PAPIIT grant IN100217, CONACyT grants F-252167 and 278017, PIIF grant and the TUM August-Wilhelm Scheer Program. The work of P.V. is supported by the Deutsche Forschungsgemeinschaft (SFB1258). 


\section{A Remarks}

In this appendix, we comment on some inaccuracies in the literature on modular symmetries in model building. First, it is important to note that the modular $\mathrm{S}$ transformation is, in general, not of order 2 , even though $\mathrm{S}^{2}$ acts trivially on $\tau$. In detail, from eq. (2.3) we get

$$
\begin{aligned}
& \tau \stackrel{\mathrm{S}}{\longmapsto}-\frac{1}{\tau}, \quad \psi \stackrel{\mathrm{S}}{\longmapsto}(-\tau)^{-k} \rho(\mathrm{S}) \psi \quad \text { for } \quad \mathrm{S}=\left(\begin{array}{cc}
0 & 1 \\
-1 & 0
\end{array}\right), \\
& \tau \stackrel{\mathrm{S}^{-1}}{\longmapsto}-\frac{1}{\tau}, \quad \psi \stackrel{\mathrm{S}^{-1}}{\longmapsto}(+\tau)^{-k} \rho(\mathrm{S})^{-1} \psi \quad \text { for } \quad \mathrm{S}^{-1}=\left(\begin{array}{cc}
0 & -1 \\
1 & 0
\end{array}\right) .
\end{aligned}
$$

Then, $\mathrm{S}^{2}$ acts as

$$
\begin{aligned}
& \tau \stackrel{\mathrm{S}}{\longmapsto}-\frac{1}{\tau} \stackrel{\mathrm{S}}{\longmapsto} \tau, \\
& \psi \stackrel{\mathrm{S}}{\longmapsto}(-\tau)^{-k} \rho(\mathrm{S}) \psi \stackrel{\mathrm{S}}{\longmapsto}\left(\frac{1}{\tau}\right)^{-k}(-\tau)^{-k} \rho(\mathrm{S})^{2} \psi=(-1)^{-k} \rho(\mathrm{S})^{2} \psi,
\end{aligned}
$$

while

$$
\begin{aligned}
& \tau \stackrel{\mathrm{S}}{\longmapsto}-\frac{1}{\tau} \stackrel{\mathrm{S}^{-1}}{\longmapsto} \tau, \\
& \psi \stackrel{\mathrm{S}}{\longmapsto}(-\tau)^{-k} \rho(\mathrm{S}) \psi \stackrel{\mathrm{S}^{-1}}{\longmapsto}\left(\frac{1}{\tau}\right)^{-k}(\tau)^{-k} \rho(\mathrm{S}) \rho\left(\mathrm{S}^{-1}\right) \psi=\psi,
\end{aligned}
$$

as expected. Let us compare eq. (A.2b) and eq. (A.3b) in some detail. In eq. (A.2b), the transformation by $\rho(\mathrm{S})^{2}$ is trivial for $\Gamma_{N}$ but can be nontrivial for $\Gamma_{N}^{\prime}$. Moreover, the factor $(-1)^{-k}$ is nontrivial for a general modular weight $k \in \mathbb{Q}$ that is not even. Consequently, we see that in general $\mathrm{S}$ and $\mathrm{S}^{-1}$ are different transformations for matter fields, even though they act identically on the $\tau$ modulus.

\section{B Explicit examples}

\section{B.1 Traditional flavor symmetry $Q_{8}$}

Let us consider the traditional flavor symmetry $Q_{8}$ of order 8 (GAP ID [8,4]). We choose the irreducible two-dimensional representation of $Q_{8}$ given by [58]

$$
\rho(\mathrm{A})=\left(\begin{array}{cc}
\mathrm{i} & 0 \\
0 & -\mathrm{i}
\end{array}\right), \quad \rho(\mathrm{B})=\left(\begin{array}{cc}
0 & \mathrm{i} \\
\mathrm{i} & 0
\end{array}\right) .
$$

The full automorphism group of $Q_{8}$ is $S_{4}$ with 24 elements. Out of these, we can identify two outer automorphisms that generate the finite modular group $S_{3}$, i.e.

$$
\begin{array}{ll}
u_{\mathrm{S}}(\mathrm{A})=\mathrm{A}^{3}, & u_{\mathrm{T}}(\mathrm{A})=\mathrm{B}, \\
u_{\mathrm{S}}(\mathrm{B})=\mathrm{AB}, & u_{\mathrm{T}}(\mathrm{B})=\mathrm{A} .
\end{array}
$$


Then, there are two choices of matrices $\rho(\mathrm{S})$ and $\rho(\mathrm{T})$ that realize these automorphisms via eq. (2.12) and generate $S_{3}$, being

$$
\rho(\mathrm{S})=\alpha\left(\begin{array}{cc}
0 & \exp ^{2 \pi \mathrm{i} / 8} \\
\exp -2 \pi \mathrm{i} / 8 & 0
\end{array}\right), \quad \rho(\mathrm{T})=\frac{\alpha}{\sqrt{2}}\left(\begin{array}{cc}
-1 & -1 \\
-1 & 1
\end{array}\right),
$$

for $\alpha= \pm 1$. For both choices of $\alpha, \rho(\mathrm{S})$ and $\rho(\mathrm{T})$ build a doublet of $S_{3}$. Moreover, the eclectic flavor group, generated by $\rho(\mathrm{A}), \rho(\mathrm{B}), \rho(\mathrm{S})$ and $\rho(\mathrm{T})$, turns out to be GL $(2,3)$. It is interesting to note that the $Q_{8}$ traditional flavor symmetry does not allow for an eclectic extension with $\mathcal{C P}$.

\section{B.2 Traditional flavor symmetry $\mathbb{Z}_{3} \times \mathbb{Z}_{3}$}

We choose a (reducible) three-dimensional representation of $\mathbb{Z}_{3} \times \mathbb{Z}_{3}$ given by

$$
\rho(\mathrm{A})=\left(\begin{array}{ccc}
\omega & 0 & 0 \\
0 & 1 & 0 \\
0 & 0 & \omega^{2}
\end{array}\right), \quad \rho(\mathrm{B})=\left(\begin{array}{ccc}
1 & 0 & 0 \\
0 & \omega & 0 \\
0 & 0 & \omega^{2}
\end{array}\right) .
$$

The full automorphism group of $\mathbb{Z}_{3} \times \mathbb{Z}_{3}$ is GL(2,3) with 48 elements. Since the group of inner automorphisms of $\mathbb{Z}_{3} \times \mathbb{Z}_{3}$ is trivial, all elements of $\operatorname{GL}(2,3)$ are outer automorphisms. It turns out that there are two classes of outer automorphisms that generate finite modular groups, either without or with $\mathcal{C P}$ :

i) Without $\mathcal{C P}$, we can choose the outer automorphisms

$$
\begin{array}{ll}
u_{\mathrm{S}}(\mathrm{A})=\mathrm{A}^{2}, & u_{\mathrm{T}}(\mathrm{A})=\mathrm{B}, \\
u_{\mathrm{S}}(\mathrm{B})=\mathrm{A}^{2} \mathrm{~B}, & u_{\mathrm{T}}(\mathrm{B})=\mathrm{A},
\end{array}
$$

which generate the finite modular group $S_{3}$. One possibility to realize these outer automorphisms via conjugation with matrices $\rho(\mathrm{S})$ and $\rho(\mathrm{T})$ is given by the choice

$$
\rho(\mathrm{S})=\left(\begin{array}{ccc}
0 & 0 & -\mathrm{i} \\
0 & -1 & 0 \\
\mathrm{i} & 0 & 0
\end{array}\right), \quad \rho(\mathrm{T})=\left(\begin{array}{ccc}
0 & -1 & 0 \\
-1 & 0 & 0 \\
0 & 0 & -1
\end{array}\right)
$$

see eq. (2.12). This three-dimensional representation of $S_{3}$ decomposes into a $\mathbf{2} \oplus \mathbf{1}^{\prime}$. Interestingly, the eclectic flavor group, generated by $\rho(\mathrm{A}), \rho(\mathrm{B}), \rho(\mathrm{S})$ and $\rho(\mathrm{T})$, is $\Delta(54)$ and the representation eq. (B.4) and eq. (B.6) is three-dimensional.

ii) Moreover, when combining eq. (B.5) with the outer automorphism

$$
u_{\mathrm{K}_{*}}(\mathrm{~A})=\mathrm{A}^{2}, \quad u_{\mathrm{K}_{*}}(\mathrm{~B})=\mathrm{B}^{2}
$$

we see that the $\mathcal{C P}$-enhanced finite modular group is $\Gamma_{2}^{*} \cong S_{3} \times \mathbb{Z}_{2}$, where $\mathrm{K}_{*}$ can be represented as

$$
\rho\left(\mathrm{K}_{*}\right)=\left(\begin{array}{ccc}
1 & 0 & 0 \\
0 & 1 & 0 \\
0 & 0 & -1
\end{array}\right)
$$


which satisfies eq. (2.24). The action on the matter fields $(\psi, \bar{\psi})^{T}$ is realized by rewriting all modular generators in the six-dimensional representation, as in eq. (4.10). In this six-dimensional representation, one can easily confirm using GAP that the eclectic flavor group including $\mathcal{C P}$ is $[108,17]$.

\section{B.3 Traditional flavor symmetry $A_{4}$}

The generators of the traditional flavor symmetry $A_{4}$ (GAP ID $[12,3]$ ) can be given in the triplet representation by the matrices

$$
\rho(\mathrm{A})=\left(\begin{array}{ccc}
1 & 0 & 0 \\
0 & -1 & 0 \\
0 & 0 & -1
\end{array}\right), \quad \rho(\mathrm{B})=\left(\begin{array}{lll}
0 & 1 & 0 \\
0 & 0 & 1 \\
1 & 0 & 0
\end{array}\right) .
$$

The full automorphism group of $A_{4}$ is $S_{4}$, which contains only two finite modular groups generated by the outer automorphisms:

i) Two outer automorphisms that generate the finite modular group $\Gamma_{2} \cong S_{3}$ are

$$
\begin{array}{ll}
u_{\mathrm{S}}(\mathrm{A})=\mathrm{B}^{2} \mathrm{AB}, & u_{\mathrm{T}}(\mathrm{A})=\mathrm{B} \mathrm{A} \mathrm{B}^{2}, \\
u_{\mathrm{S}}(\mathrm{B})=\mathrm{B}^{2}, & u_{\mathrm{T}}(\mathrm{B})=\mathrm{B}^{2} .
\end{array}
$$

In the representation (B.9), these automorphisms can be expressed as

$$
\rho(\mathrm{S})=\left(\begin{array}{lll}
0 & 1 & 0 \\
1 & 0 & 0 \\
0 & 0 & 1
\end{array}\right), \quad \rho(\mathrm{T})=\left(\begin{array}{lll}
0 & 0 & 1 \\
0 & 1 & 0 \\
1 & 0 & 0
\end{array}\right) .
$$

The generators $\rho(\mathrm{A}), \rho(\mathrm{B}), \rho(\mathrm{S})$ and $\rho(\mathrm{T})$ generate the eclectic flavor group $S_{4}$, which is isomorphic to the full automorphism group $S_{4}$.

ii) Two outer automorphisms that generate the finite modular group $\Gamma_{4} \cong S_{4}$ are

$$
\begin{array}{ll}
u_{\mathrm{S}}(\mathrm{A})=\mathrm{B}^{2} \mathrm{AB}, & u_{\mathrm{T}}(\mathrm{A})=\mathrm{B} \mathrm{A} \mathrm{B}^{2}, \\
u_{\mathrm{S}}(\mathrm{B})=\mathrm{B}^{2}, & u_{\mathrm{T}}(\mathrm{B})=\mathrm{B} \mathrm{A} \mathrm{B} .
\end{array}
$$

In the representation (B.9), these automorphisms can be expressed as

$$
\rho(\mathrm{S})=\left(\begin{array}{lll}
0 & 1 & 0 \\
1 & 0 & 0 \\
0 & 0 & 1
\end{array}\right), \quad \rho(\mathrm{T})=\left(\begin{array}{ccc}
0 & 0 & 1 \\
0 & -1 & 0 \\
-1 & 0 & 0
\end{array}\right),
$$

and the eclectic extension of the traditional flavor symmetry $A_{4}$ by the $\Gamma_{4} \cong S_{4}$ finite modular group yields the eclectic flavor group $S_{4}$.

Let us conclude this example with two remarks, both related to the fact that the group of outer automorphisms of $A_{4}$ is $\mathbb{Z}_{2}$, i.e. very small. First, the $A_{4}$ traditional flavor group does not allow for an eclectic flavor group with $\mathcal{C P}$. Secondly, for both eclectic extensions of the traditional flavor group $A_{4}$ by either by $S_{3}$ or $S_{4}$ the eclectic flavor group is $S_{4}$. Note that, even though the eclectic flavor groups are identical (i.e. $S_{4}$ ), the eclectic extensions of $A_{4}$ by $S_{3}$ or $S_{4}$ yield different theories, since, for example, Yukawa couplings have to be modular forms of either $S_{3}$ or $S_{4}$. 


\section{B.4 Traditional flavor symmetry $T^{\prime}$}

The generators of the traditional flavor symmetry $T^{\prime}$ (GAP ID $[24,3]$ ) can be given in the $\mathbf{1} \oplus \mathbf{2}$ representation by the matrices

$$
\rho(\mathrm{A})=\left(\begin{array}{ccc}
1 & 0 & 0 \\
0 & \omega^{2} & 0 \\
0 & 0 & \omega
\end{array}\right), \rho(\mathrm{B})=\left(\begin{array}{ccc}
1 & 0 & 0 \\
0 & -1 & 0 \\
0 & 0 & -1
\end{array}\right), \rho(\mathrm{C})=\frac{-\mathrm{i}}{\sqrt{3}}\left(\begin{array}{ccc}
\mathrm{i} \sqrt{3} & 0 & 0 \\
0 & 1 & \sqrt{2} \\
0 & \sqrt{2} & -1
\end{array}\right),
$$

where $\omega:=\exp 2 \pi \mathrm{i} / 3$. The full automorphism group of $T^{\prime}$ is $S_{4}$. One can choose the following two outer automorphisms of this group to generate the finite modular group $\Gamma_{2} \cong S_{3}$ :

$$
\begin{array}{ll}
u_{\mathrm{S}}(\mathrm{A})=\mathrm{A}^{2}, & u_{\mathrm{T}}(\mathrm{A})=\mathrm{A}^{2}, \\
u_{\mathrm{S}}(\mathrm{B})=\mathrm{B}, & u_{\mathrm{T}}(\mathrm{B})=\mathrm{B}, \\
u_{\mathrm{S}}(\mathrm{C})=\mathrm{CACA}^{2}, & u_{\mathrm{T}}(\mathrm{C})=\mathrm{CB} .
\end{array}
$$

In the representation (B.14), these automorphisms are given by

$$
\rho(\mathrm{S})=\left(\begin{array}{ccc}
\alpha & 0 & 0 \\
0 & 0 & \beta \mathrm{i} \omega \\
0 & -\beta \mathrm{i} \omega^{2} & 0
\end{array}\right), \quad \rho(\mathrm{T})=\left(\begin{array}{ccc}
\alpha & 0 & 0 \\
0 & 0 & \beta \mathrm{i} \\
0 & -\beta \mathrm{i} & 0
\end{array}\right),
$$

for $\alpha= \pm 1$ and $\beta= \pm 1$. The generators $\rho(\mathrm{A}), \rho(\mathrm{B}), \rho(\mathrm{C}), \rho(\mathrm{S})$ and $\rho(\mathrm{T})$ generate the eclectic flavor group GL(2,3).

Note that the $T^{\prime}$ traditional flavor symmetry does not allow for an eclectic flavor group with $\mathcal{C P}$.

\section{B.5 Traditional flavor symmetry $\Delta(27)$}

Next, we choose the traditional flavor symmetry $\Delta(27)$ (GAP ID [27, 3]). It can be generated in a triplet representation by the matrices

$$
\rho(\mathrm{A})=\left(\begin{array}{lll}
0 & 1 & 0 \\
0 & 0 & 1 \\
1 & 0 & 0
\end{array}\right), \quad \rho(\mathrm{B})=\left(\begin{array}{ccc}
\omega & 0 & 0 \\
0 & 1 & 0 \\
0 & 0 & \omega^{2}
\end{array}\right), \quad \rho(\mathrm{C})=\left(\begin{array}{ccc}
\omega^{2} & 0 & 0 \\
0 & \omega & 0 \\
0 & 0 & 1
\end{array}\right),
$$

where $\rho(\mathrm{C})=\rho(\mathrm{A})^{2} \rho(\mathrm{B}) \rho(\mathrm{A})$, see ref. [58]. Out of the full group of automorphisms with GAP ID $[432,734]$ we identify two classes of outer automorphisms that generate finite modular groups.

i) We can choose

$$
\begin{array}{ll}
u_{\mathrm{S}}(\mathrm{A})=\mathrm{A}^{2}, & u_{\mathrm{T}}(\mathrm{A})=\mathrm{A}^{2}, \\
u_{\mathrm{S}}(\mathrm{B})=\mathrm{B}^{2}, & u_{\mathrm{T}}(\mathrm{B})=\mathrm{A}^{2} \mathrm{~B}^{2} \mathrm{~A} .
\end{array}
$$

These automorphisms generate $S_{3}$. Then, there are two choices of matrices that generate $S_{3}$ and realize these outer automorphisms by conjugation, eq. (2.12), being

$$
\rho(\mathrm{S})=\alpha\left(\begin{array}{lll}
0 & 0 & 1 \\
0 & 1 & 0 \\
1 & 0 & 0
\end{array}\right), \quad \rho(\mathrm{T})=\alpha\left(\begin{array}{lll}
1 & 0 & 0 \\
0 & 0 & 1 \\
0 & 1 & 0
\end{array}\right)
$$


for $\alpha= \pm 1$. In both cases, $\rho(\mathrm{S})$ and $\rho(\mathrm{T})$ generate the finite modular group $\Gamma_{2} \cong S_{3}$, where the triplet of $\Delta(27)$ builds a $\mathbf{2} \oplus \mathbf{1}$ of $S_{3}$ if $\alpha=1$, or $\mathbf{2} \oplus \mathbf{1}^{\prime}$ if $\alpha=-1$. In both cases, the eclectic flavor group is $\Delta(54)$.

Incorporating the $\mathcal{C P}$-like transformation $\mathrm{K}_{*}$ can be done by including the outer automorphism

$$
u_{\mathrm{K}_{*}}(\mathrm{~A})=\mathrm{A}, \quad u_{\mathrm{K}_{*}}(\mathrm{~B})=\mathrm{B} \mathrm{A}^{2} \mathrm{~B},
$$

which, together with eq. (B.18), leads to the $\mathcal{C} \mathcal{P}$-enhanced finite modular group $S_{3} \times \mathbb{Z}_{2}$. In the representation eq. (B.17), it can be written as

$$
\rho\left(\mathrm{K}_{*}\right)=\frac{\mathrm{i}}{\sqrt{3}}\left(\begin{array}{ccc}
1 & \omega^{2} & \omega^{2} \\
\omega^{2} & 1 & \omega^{2} \\
\omega^{2} & \omega^{2} & 1
\end{array}\right)
$$

which satisfies eq. $(2.24)$. The action on the matter fields $(\psi, \bar{\psi})^{T}$ is realized by rewriting all modular generators in the six-dimensional representation, as in eq. (4.10). In this case, the eclectic flavor group including $\mathcal{C P}$ is $[108,17]$.

ii) Furthermore, we can choose

$$
\begin{array}{ll}
u_{\mathrm{S}}(\mathrm{A})=\mathrm{B}^{2} \mathrm{~A}, & u_{\mathrm{T}}(\mathrm{A})=\mathrm{B} \mathrm{A}, \\
u_{\mathrm{S}}(\mathrm{B})=(\mathrm{AB})^{2}, & u_{\mathrm{T}}(\mathrm{B})=\mathrm{B} .
\end{array}
$$

These outer automorphisms generate $T^{\prime}$. Then, there are three choices of matrices that generate $T^{\prime}$ and realize these automorphisms by conjugation, being

$$
\rho(\mathrm{S})=-\frac{\mathrm{i}}{\sqrt{3}}\left(\begin{array}{ccc}
1 & \omega & \omega \\
\omega^{2} & \omega & \omega^{2} \\
\omega^{2} & \omega^{2} & \omega
\end{array}\right), \quad \rho(\mathrm{T})=\omega^{k}\left(\begin{array}{ccc}
1 & 0 & 0 \\
0 & \omega^{2} & 0 \\
0 & 0 & \omega^{2}
\end{array}\right)
$$

for $k=0,1,2$. In these cases, the eclectic flavor group results in $\Omega(1$ ) (with GAP ID $[648,533])$.

Incorporating the $\mathcal{C P}$-like transformation $\mathrm{K}_{*}$ can be done by including the outer automorphism

$$
u_{\mathrm{K}_{*}}(\mathrm{~A})=\mathrm{A}^{2} \mathrm{~B}, \quad u_{\mathrm{K}_{*}}(\mathrm{~B})=\mathrm{A}^{2} \mathrm{~B} \mathrm{~A}
$$

which, together with eq. (B.22), leads to the $\mathcal{C P}$-enhanced finite modular group $\mathrm{GL}(2,3)$. In the representation eq. (B.17), it can be written as

$$
\rho\left(\mathrm{K}_{*}\right)=e^{\mathrm{i} \gamma}\left(\begin{array}{ccc}
\omega^{2} & 0 & 0 \\
0 & 0 & 1 \\
0 & 1 & 0
\end{array}\right)
$$

which satisfies eq. (2.24) with $\gamma \in \mathbb{R}$. The action on the matter fields $(\psi, \bar{\psi})^{T}$ is realized by rewriting all modular generators in the six-dimensional representation, as in eq. (4.10). The resulting eclectic flavor group is [1296, 2891]. 


\section{B.6 Trivial extension of the traditional flavor symmetry $\Delta(54)$}

As discussed before section 2.1, inner automorphisms can only produce a trivial extension of a traditional flavor symmetry. To illustrate this scenario, let us entertain the possibility of $\Delta(54)$ being extended by a $\Gamma_{2} \cong S_{3}$ finite modular symmetry generated by the $\Delta(54)$ inner automorphisms

$$
\begin{array}{ll}
u_{\mathrm{S}}(\mathrm{A})=\mathrm{A}^{2}, & u_{\mathrm{T}}(\mathrm{A})=\mathrm{A}^{2}, \\
u_{\mathrm{S}}(\mathrm{B})=\mathrm{B}^{2}, & u_{\mathrm{T}}(\mathrm{B})=\mathrm{A}^{2} \mathrm{~B}^{2} \mathrm{~A}, \\
u_{\mathrm{S}}(\mathrm{C})=\mathrm{C}, & u_{\mathrm{T}}(\mathrm{C})=\mathrm{AC} ;
\end{array}
$$

see section 4. For the three-dimensional representation eq. (4.2) of $\Delta(54)$, these are given in terms of eq. (2.12) by the matrix representations

$$
\rho(\mathrm{S})=-\left(\begin{array}{lll}
1 & 0 & 0 \\
0 & 0 & 1 \\
0 & 1 & 0
\end{array}\right) \quad \text { and } \quad \rho(\mathrm{T})=-\left(\begin{array}{lll}
0 & 1 & 0 \\
1 & 0 & 0 \\
0 & 0 & 1
\end{array}\right) .
$$

From eq. (2.6), one can easily see that $\rho(\mathrm{S})$ and $\rho(\mathrm{T})$ generate the finite modular group $\Gamma_{2} \cong S_{3}$. Notice however that $\rho(\mathrm{S})=\rho(\mathrm{C})$ and $\rho(\mathrm{T})=\rho(\mathrm{A})^{2} \rho(\mathrm{C})$. As in eq. (2.17), we observe here that the action of the $S_{3}$ modular generators is compensated by the $\Delta(54)$ elements represented by $\rho(\mathrm{C})^{-1}$ and $\rho\left(\mathrm{A}^{2} \mathrm{C}\right)^{-1}$. Therefore, the action of the finite modular group $S_{3}$ based on inner automorphisms can be given by the trivial representation $\rho(\gamma)=\mathbb{1}$, which amounts to a trivial extension of the traditional flavor symmetry.

As a side remark, a careful inspection reveals that there are no outer automorphisms of $\Delta(54)$ that generate a $\Gamma_{2}$ finite modular group and simultaneously satisfy eqs. (2.6) and (2.12). Thus, the $\Delta(54)$ traditional flavor group cannot be enhanced nontrivially by a $\Gamma_{2}$ finite modular group.

Open Access. This article is distributed under the terms of the Creative Commons Attribution License (CC-BY 4.0), which permits any use, distribution and reproduction in any medium, provided the original author(s) and source are credited.

\section{References}

[1] F. Feruglio and A. Romanino, Neutrino Flavour Symmetries, arXiv:1912.06028 [INSPIRE].

[2] F. Feruglio, Are neutrino masses modular forms?, in From My Vast Repertoire ...: Guido Altarelli's Legacy, A. Levy, S. Forte and G. Ridolfi eds., pp. 227-266 (2019) [DOI] [arXiv: 1706.08749] [INSPIRE].

[3] G. Altarelli and F. Feruglio, Tri-bimaximal neutrino mixing, $A_{4}$ and the modular symmetry, Nucl. Phys. B 741 (2006) 215 [hep-ph/0512103] [INSPIRE].

[4] R. de Adelhart Toorop, F. Feruglio and C. Hagedorn, Finite Modular Groups and Lepton Mixing, Nucl. Phys. B 858 (2012) 437 [arXiv:1112.1340] [INSPIRE].

[5] T. Kobayashi, K. Tanaka and T.H. Tatsuishi, Neutrino mixing from finite modular groups, Phys. Rev. D 98 (2018) 016004 [arXiv:1803.10391] [INSPIRE]. 
[6] J.T. Penedo and S.T. Petcov, Lepton Masses and Mixing from Modular $S_{4}$ Symmetry, Nucl. Phys. B 939 (2019) 292 [arXiv:1806.11040] [INSPIRE].

[7] J.C. Criado and F. Feruglio, Modular Invariance Faces Precision Neutrino Data, SciPost Phys. 5 (2018) 042 [arXiv:1807.01125] [INSPIRE].

[8] T. Kobayashi, N. Omoto, Y. Shimizu, K. Takagi, M. Tanimoto and T.H. Tatsuishi, Modular $A_{4}$ invariance and neutrino mixing, JHEP 11 (2018) 196 [arXiv:1808.03012] [INSPIRE].

[9] P.P. Novichkov, J.T. Penedo, S.T. Petcov and A.V. Titov, Modular $S_{4}$ models of lepton masses and mixing, JHEP 04 (2019) 005 [arXiv: 1811.04933] [INSPIRE].

[10] P.P. Novichkov, J.T. Penedo, S.T. Petcov and A.V. Titov, Modular $A_{5}$ symmetry for flavour model building, JHEP 04 (2019) 174 [arXiv: 1812.02158] [INSPIRE].

[11] F.J. de Anda, S.F. King and E. Perdomo, SU(5) Grand Unified Theory with $A_{4}$ Modular Symmetry, Phys. Rev. D 101 (2020) 015028 [arXiv:1812.05620] [InSPIRE].

[12] H. Okada and M. Tanimoto, CP violation of quarks in $A_{4}$ modular invariance, Phys. Lett. $\mathbf{B}$ 791 (2019) 54 [arXiv:1812.09677] [INSPIRE].

[13] T. Kobayashi, Y. Shimizu, K. Takagi, M. Tanimoto, T.H. Tatsuishi and H. Uchida, Finite modular subgroups for fermion mass matrices and baryon/lepton number violation, Phys. Lett. B 794 (2019) 114 [arXiv:1812.11072] [INSPIRE].

[14] P.P. Novichkov, S.T. Petcov and M. Tanimoto, Trimaximal Neutrino Mixing from Modular $A_{4}$ Invariance with Residual Symmetries, Phys. Lett. B 793 (2019) 247 [arXiv:1812.11289] [INSPIRE].

[15] G.-J. Ding, S.F. King and X.-G. Liu, Neutrino mass and mixing with $A_{5}$ modular symmetry, Phys. Rev. D 100 (2019) 115005 [arXiv:1903.12588] [INSPIRE].

[16] T. Nomura and H. Okada, A modular $A_{4}$ symmetric model of dark matter and neutrino, Phys. Lett. B 797 (2019) 134799 [arXiv: 1904.03937] [INSPIRE].

[17] P.P. Novichkov, J.T. Penedo, S.T. Petcov and A.V. Titov, Generalised CP Symmetry in Modular-Invariant Models of Flavour, JHEP 07 (2019) 165 [arXiv: 1905.11970] [INSPIRE].

[18] H. Okada and M. Tanimoto, Towards unification of quark and lepton flavors in $A_{4}$ modular invariance, arXiv:1905.13421 [INSPIRE].

[19] I. De Medeiros Varzielas, S.F. King and Y.-L. Zhou, Multiple modular symmetries as the origin of flavour, arXiv: 1906.02208 [INSPIRE].

[20] T. Nomura and H. Okada, A two loop induced neutrino mass model with modular $A_{4}$ symmetry, arXiv:1906.03927 [INSPIRE].

[21] T. Kobayashi, Y. Shimizu, K. Takagi, M. Tanimoto and T.H. Tatsuishi, Modular $S_{3}$ invariant flavor model in SU(5) GUT, arXiv:1906.10341 [INSPIRE].

[22] X.-G. Liu and G.-J. Ding, Neutrino Masses and Mixing from Double Covering of Finite Modular Groups, JHEP 08 (2019) 134 [arXiv:1907.01488] [INSPIRE].

[23] H. Okada and Y. Orikasa, Modular $S_{3}$ symmetric radiative seesaw model, Phys. Rev. D 100 (2019) 115037 [arXiv:1907.04716] [INSPIRE].

[24] T. Kobayashi, Y. Shimizu, K. Takagi, M. Tanimoto and T.H. Tatsuishi, New $A_{4}$ lepton flavor model from $S_{4}$ modular symmetry, arXiv:1907.09141 [INSPIRE]. 
[25] G.-J. Ding, S.F. King and X.-G. Liu, Modular $A_{4}$ symmetry models of neutrinos and charged leptons, JHEP 09 (2019) 074 [arXiv:1907.11714] [INSPIRE].

[26] H. Okada and Y. Orikasa, A radiative seesaw model in modular $A_{4}$ symmetry, arXiv: 1907.13520 [INSPIRE].

[27] S.F. King and Y.-L. Zhou, Trimaximal TM $M_{1}$ mixing with two modular $S_{4}$ groups, Phys. Rev. D 101 (2020) 015001 [arXiv: 1908.02770] [INSPIRE].

[28] T. Nomura, H. Okada and O. Popov, A modular $A_{4}$ symmetric scotogenic model, arXiv: 1908.07457 [INSPIRE].

[29] H. Okada and Y. Orikasa, Neutrino mass model with a modular $S_{4}$ symmetry, arXiv: 1908.08409 [INSPIRE].

[30] J.C. Criado, F. Feruglio, F. Feruglio and S.J.D. King, Modular Invariant Models of Lepton Masses at Levels 4 and 5, arXiv:1908.11867 [INSPIRE].

[31] T. Kobayashi, Y. Shimizu, K. Takagi, M. Tanimoto and T.H. Tatsuishi, A4 lepton flavor model and modulus stabilization from $S_{4}$ modular symmetry, Phys. Rev. D 100 (2019) 115045 [arXiv: 1909.05139] [INSPIRE].

[32] T. Asaka, Y. Heo, T.H. Tatsuishi and T. Yoshida, Modular $A_{4}$ invariance and leptogenesis, JHEP 01 (2020) 144 [arXiv:1909.06520] [INSPIRE].

[33] M.-C. Chen, S. Ramos-Sánchez and M. Ratz, A note on the predictions of models with modular flavor symmetries, Phys. Lett. B 801 (2020) 135153 [arXiv: 1909.06910] [INSPIRE].

[34] G.-J. Ding, S.F. King, X.-G. Liu and J.-N. Lu, Modular $S_{4}$ and $A_{4}$ symmetries and their fixed points: new predictive examples of lepton mixing, JHEP 12 (2019) 030 [arXiv: 1910.03460] [INSPIRE].

[35] D. Zhang, A modular $A_{4}$ symmetry realization of two-zero textures of the Majorana neutrino mass matrix, Nucl. Phys. B 952 (2020) 114935 [arXiv: 1910.07869] [InSPIRE].

[36] X. Wang and S. Zhou, The Minimal Seesaw Model with a Modular $S_{4}$ Symmetry, arXiv: 1910.09473 [INSPIRE].

[37] T. Kobayashi, Y. Shimizu, K. Takagi, M. Tanimoto, T.H. Tatsuishi and H. Uchida, $C P$ violation in modular invariant flavor models, arXiv: 1910.11553 [INSPIRE].

[38] T. Nomura, H. Okada and S. Patra, An Inverse Seesaw model with $A_{4}$-modular symmetry, arXiv: 1912.00379 [INSPIRE].

[39] T. Kobayashi, T. Nomura and T. Shimomura, Type II seesaw models with modular $A_{4}$ symmetry, arXiv:1912.00637 [INSPIRE].

[40] J.-N. Lu, X.-G. Liu and G.-J. Ding, Modular symmetry origin of texture zeros and quark lepton unification, arXiv: 1912.07573 [INSPIRE].

[41] X. Wang, Lepton Flavor Mixing and CP-violation in the Minimal Type-(I+II) Seesaw Model with a Modular $A_{4}$ Symmetry, arXiv:1912.13284 [INSPIRE].

[42] A. Baur, H.P. Nilles, A. Trautner and P.K.S. Vaudrevange, Unification of Flavor, CP and Modular Symmetries, Phys. Lett. B 795 (2019) 7 [arXiv:1901.03251] [InSPIRE].

[43] A. Baur, H.P. Nilles, A. Trautner and P.K.S. Vaudrevange, A String Theory of Flavor and CP, Nucl. Phys. B 947 (2019) 114737 [arXiv: 1908.00805] [INSPIRE]. 
[44] T. Kobayashi, S. Nagamoto and S. Uemura, Modular symmetry in magnetized/intersecting D-brane models, PTEP 2017 (2017) 023B02 [arXiv: 1608.06129] [INSPIRE].

[45] T. Kobayashi, S. Nagamoto, S. Takada, S. Tamba and T.H. Tatsuishi, Modular symmetry and non-Abelian discrete flavor symmetries in string compactification, Phys. Rev. D 97 (2018) 116002 [arXiv:1804.06644] [INSPIRE].

[46] T. Kobayashi and S. Tamba, Modular forms of finite modular subgroups from magnetized D-brane models, Phys. Rev. D 99 (2019) 046001 [arXiv:1811.11384] [INSPIRE].

[47] Y. Kariyazono, T. Kobayashi, S. Takada, S. Tamba and H. Uchida, Modular symmetry anomaly in magnetic flux compactification, Phys. Rev. D 100 (2019) 045014 [arXiv: 1904.07546] [INSPIRE].

[48] T. Kobayashi, H.P. Nilles, F. Ploger, S. Raby and M. Ratz, Stringy origin of non-Abelian discrete flavor symmetries, Nucl. Phys. B 768 (2007) 135 [hep-ph/0611020] [INSPIRE].

[49] S. Groot Nibbelink and P.K.S. Vaudrevange, T-duality orbifolds of heterotic Narain compactifications, JHEP 04 (2017) 030 [arXiv: 1703.05323] [INSPIRE].

[50] The GAP Group, GAP - Groups, Algorithms, and Programming, Version 4.10.2, (2019).

[51] J.-P. Serre, Trees, Springer Berlin Heidelberg (1980).

[52] L.J. Dixon, V. Kaplunovsky and J. Louis, On Effective Field Theories Describing $(2,2)$ Vacua of the Heterotic String, Nucl. Phys. B 329 (1990) 27 [inSPIRE].

[53] L.E. Ibáñez and D. Lüst, Duality anomaly cancellation, minimal string unification and the effective low-energy Lagrangian of 4-D strings, Nucl. Phys. B 382 (1992) 305 [hep-th/9202046] [INSPIRE].

[54] Y. Olguín-Trejo and S. Ramos-Sánchez, Kähler potential of heterotic orbifolds with multiple Kähler moduli, J. Phys. Conf. Ser. 912 (2017) 012029 [arXiv: 1707.09966] [InSPIRE].

[55] F. Feruglio, C. Hagedorn and R. Ziegler, Lepton Mixing Parameters from Discrete and CP Symmetries, JHEP 07 (2013) 027 [arXiv: 1211.5560] [INSPIRE].

[56] M. Holthausen, M. Lindner and M.A. Schmidt, CP and Discrete Flavour Symmetries, JHEP 04 (2013) 122 [arXiv: 1211.6953] [INSPIRE].

[57] M.-C. Chen, M. Fallbacher, K.T. Mahanthappa, M. Ratz and A. Trautner, CP Violation from Finite Groups, Nucl. Phys. B 883 (2014) 267 [arXiv:1402.0507] [InSPIRE].

[58] H. Ishimori, T. Kobayashi, H. Ohki, Y. Shimizu, H. Okada and M. Tanimoto, Non-Abelian Discrete Symmetries in Particle Physics, Prog. Theor. Phys. Suppl. 183 (2010) 1 [arXiv: 1003.3552] [INSPIRE].

[59] T. Dent, CP violation and modular symmetries, Phys. Rev. D 64 (2001) 056005 [hep-ph/0105285] [INSPIRE].

[60] J.A. Escobar and C. Luhn, The Flavor Group $\Delta\left(6 n^{2}\right)$, J. Math. Phys. 50 (2009) 013524 [arXiv:0809.0639] [INSPIRE].

[61] C.S. Lam, The Unique Horizontal Symmetry of Leptons, Phys. Rev. D 78 (2008) 073015 [arXiv:0809.1185] [INSPIRE].

[62] H. Ishimori, T. Kobayashi, H. Okada, Y. Shimizu and M. Tanimoto, Lepton Flavor Model from Delta(54) Symmetry, JHEP 04 (2009) 011 [arXiv:0811.4683] [INSPIRE]. 
[63] W. Grimus and L. Lavoura, Tri-bimaximal lepton mixing from symmetry only, JHEP 04 (2009) 013 [arXiv:0811.4766] [INSPIRE].

[64] D. Jurciukonis and L. Lavoura, GAP listing of the finite subgroups of U(3) of order smaller than 2000, PTEP 2017 (2017) 053A03 [arXiv:1702.00005] [INSPIRE].

[65] C.-Y. Yao and G.-J. Ding, Lepton and Quark Mixing Patterns from Finite Flavor Symmetries, Phys. Rev. D 92 (2015) 096010 [arXiv:1505.03798] [InSPIRE].

[66] S.F. King and P.O. Ludl, Direct and Semi-Direct Approaches to Lepton Mixing with a Massless Neutrino, JHEP 06 (2016) 147 [arXiv: 1605.01683] [INSPIRE].

[67] C. Hagedorn, A. Meroni and L. Vitale, Mixing patterns from the groups $\Sigma(n \phi)$, J. Phys. A 47 (2014) 055201 [arXiv:1307.5308] [INSPIRE].

[68] F. Beye, T. Kobayashi and S. Kuwakino, Gauge Origin of Discrete Flavor Symmetries in Heterotic Orbifolds, Phys. Lett. B 736 (2014) 433 [arXiv:1406.4660] [InSPIRE].

[69] H.P. Nilles, M. Ratz, A. Trautner and P.K.S. Vaudrevange, CP violation from string theory, Phys. Lett. B 786 (2018) 283 [arXiv:1808.07060] [InSPIRE].

[70] H.P. Nilles et al., work in progress. 